\title{
Synthesis and Characterization of Bola-type
}

\section{Amphiphilic Dendritic Macromolecules}

Lovorka Degoricija, Michael A. Carnahan ${ }^{\dagger}$, C. Starck Johnson, ${ }^{\jmath}$ Terry Kim, ${ }^{\jmath}$ and Mark W. Grinstaff*

Departments of Chemistry and Biomedical Engineering

Boston University, Metcalf Center for Science and Engineering

590 Commonwealth Avenue

Boston, MA, 02215

Tel: (617) 358-3429

Fax: (617) 353-6466

email: mgrin@bu.edu

$\dagger$ Department of Chemistry, Duke University.

${ }^{\text {` }}$ Duke University Eye Center, Durham, North Carolina

All solvents were dried using a solvent column prior to use (DCM, THF). All chemicals were purchased from Aldrich and Lancaster and used without further purification (DCC 99\%, DMAP 99\%) with the exception of methacrylic anhydride, which was distilled prior to use. Poly(ethylene glycol), PEG (average Mw 3400, 10000, and 20000), was dried under vacuum at $120^{\circ} \mathrm{C}$ for three hours before use in coupling reactions. All reactions were performed under a nitrogen atmosphere and at room temperature unless 
otherwise noted. NMR spectra were determined by Varian INOVA spectrometer at $400 \mathrm{MHz}$ for ${ }^{1} \mathrm{H}$ and $100 \mathrm{MHz}$ for ${ }^{13} \mathrm{C}$. Fast atom bombardment mass spectra (FAB-MS) were obtained on a JEOL JMSSX102A spectrometer using a 3-nitrobenzyl alcohol matrix. MALDI-MS was performed by PerSeptive Biosystems Voyager-DE BioSpectrometer Workstation using 2-(4-hydroxyphenylazo)-benzoic acid (HABA). Elemental analysis was obtained from Atlantic Microlab, Inc. Size exclusion chromatography (SEC) data was obtained using a Polymer Laboratories PLgel $3 \mu \mathrm{m}$ MIXED-E column with THF as the eluent and a Rainin HPLC system (temperature $25{ }^{\circ} \mathrm{C}$; flow rate $=1.0 \mathrm{~mL} / \mathrm{min}$ ). Poly (ethylene glycol) standards $\mathrm{Mw}=1500,4450,6240,12000$, and 23000 were used for calibration purposes. A TA instruments differential scanning calorimetery, DSC $Q_{100}$, modulated DSC was used to collect the $\mathrm{T}_{\mathrm{m}}$ or $\mathrm{T}_{\mathrm{g}}$ (equilibrate to $-60{ }^{\circ} \mathrm{C}$, ramp $3.00{ }^{\circ} \mathrm{C} / \min$ to $200{ }^{\circ} \mathrm{C}$, modulate $\pm 1.00{ }^{\circ} \mathrm{C}$ every $40 \mathrm{~s}$, isothermal for $5 \mathrm{~min}$, repeat procedure 2 more times). $\mathrm{DCM}=$ dichloromethane, $\mathrm{THF}=$ tetrahydrofuran, $\mathrm{DCC}=$ dicyclohexylcarbodiimide, $\mathrm{DMAP}=4$-(dimethylamino)pyridine, $\mathrm{DCU}=1,3$-dicyclohexylurea, $\mathrm{Pd} / \mathrm{C}=$ $10 \%$ palladium con activated carbon.

Synthesis of cis-1,3-O -benzylidine glycerol-Glycerol (627.4 g, $6.33 \mathrm{~mol})$ was dissolved in benzaldehyde $(615 \mathrm{~mL}, 6.05 \mathrm{~mol})$ along with a catalytic amount of sulfuric acid $(0.5 \mathrm{~mL})$ and allowed to stir for 24 hours. The reaction was heated at $40{ }^{\circ} \mathrm{C}$ under reduced pressure for 4 hours to azeotropically remove water. After 4 hours, the flask was cooled to room temperature and stirred overnight. Ethyl ether $(900 \mathrm{~mL})$ was added to the cooled reaction mixture and was washed with a $10 \%$ solution of sodium carbonate $(2 \times 300 \mathrm{~mL})$ and brine $(300 \mathrm{~mL})$. The organic phase was dried with sodium sulfate, filtered and placed in a freezer $\left(-20^{\circ} \mathrm{C}\right)$ overnight. The solid was vacuum filtered and re-dissolved in ethyl ether $(3 \mathrm{x} 1$ L) and placed in a freezer for 4 hours. The mother liquor was reduced under vacuum and was diluted by adding glycerol $(50 \mathrm{~mL})$ and sulfuric acid $(0.5 \mathrm{~mL})$ and stirred for 4 hours at room temperature. Ethyl ether $(900 \mathrm{~mL})$ was added and the aqueous phase was extracted with $10 \%$ sodium carbonate solution $(2 \mathrm{x}$ $200 \mathrm{~mL})$ and brine $(200 \mathrm{~mL})$. The organic phase was dried with sodium sulfate and placed in a freezer for 24 hours. The precipitate was filtered and re-crystallized three more times in ethyl ether (1L) (10\% yield). ${ }^{1} \mathrm{H}$ NMR $\left(\mathrm{CDCl}_{3}, 400 \mathrm{MHz}\right): \delta 3.08\left(\mathrm{~d}, 2,-\mathrm{CH}_{2}-\right), 3.62\left(\mathrm{~d}, 2,-\mathrm{CH}_{2}-\right), 4.18(\mathrm{~m}, 1,-\mathrm{CH}), 7.24(\mathrm{~m}, 5,-\mathrm{CH})$. 
${ }^{13} \mathrm{C}$ NMR $\left(\mathrm{CDCl}_{3}, 100 \mathrm{MHz}\right): \delta 138.08$ C), $129.29(\underline{\mathrm{CH}}), 128.52(\underline{\mathrm{CH}}), 126.12(\underline{\mathrm{CH}}), 101.83(\underline{\mathrm{C}} \mathrm{H})$, $72.47(\underline{\mathrm{CH}}), 64.17\left(\underline{\mathrm{CH}}_{2}\right)$. GC-MS: $179 \mathrm{~m} / \mathrm{z}[\mathrm{M}-\mathrm{H}]^{+}$(Theory: $\left.180.2 \mathrm{~m} / \mathrm{z}[\mathrm{M}]^{+}\right)$

Synthesis of 2-(cis-1,2-O-benzylidene glycerol)succinic acid monoester-To cis-1,3-Obenzylidene glycerol (50.0 g, $277 \mathrm{mmol})$, succinic acid $(35.06 \mathrm{~g}, 350 \mathrm{mmol})$ was added and the mixture was dissolved in pyridine $(150 \mathrm{~mL}, 1.855 \mathrm{~mol})$. The entire contents were allowed to stir for 24 hours. When fully dissolved, the reaction mixture was placed in an oil bath at $40{ }^{\circ} \mathrm{C}$ under reduced pressure for 4 hours to remove the pyridine. The solid was re-dissolved in DCM (300 mL) and washed with $0.2 \mathrm{~N} \mathrm{HCl}$ (3 $3300 \mathrm{~mL})$, where the aqueous phase was kept at $\mathrm{pH}$ 1.00. A white solid was isolated after precipitation in cold $\left(-20^{\circ} \mathrm{C}\right)$ ethanol $(100 \mathrm{~mL})$. The mother liquor was reduced under pressure and the monoester was re-precipitated in cold ethanol $(50 \mathrm{~mL})(75 \%$ yield $) .{ }^{1} \mathrm{H} \mathrm{NMR}\left(\mathrm{CDCl}_{3}, 400 \mathrm{MHz}\right): \delta 2.68\left(\mathrm{~m}, 4,-\mathrm{CH}_{2}-\right.$ $\mathrm{CH}_{2}-$ ), 4.13 (m, 2, - $\left.\mathrm{CH}_{2}-\mathrm{CH}-\mathrm{CH}_{2}-\right), 4.33$ (m, 2, $\left.-\mathrm{CH}_{2}-\mathrm{CH}-\mathrm{CH}_{2}-\right), 4.70$ (m, 1, - $\left.\mathrm{CH}_{2}-\mathrm{CH}-\mathrm{CH}_{2}\right), 5.51$ (s, 1 , $\mathrm{CH}), 7.34(\mathrm{~m}, 3$, aromatic $\mathrm{CH}), 7.47(\mathrm{~m}, 2$, aromatic $\mathrm{CH}) .{ }^{13} \mathrm{C} \quad \mathrm{NMR}\left(\mathrm{CDCl}_{3}, 100 \mathrm{MHz}\right)$ :

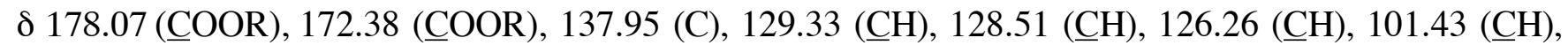
$69.15\left(\underline{\mathrm{CH}}_{2}\right), 66.57(\underline{\mathrm{C}} \mathrm{H}), 29.24\left(\underline{\mathrm{CH}}_{2}\right), 29.05\left(\underline{\mathrm{CH}}_{2}\right)$. FTIR: $v\left(\mathrm{~cm}^{-1}\right) 2931$ (aliph. C-H stretch), 1713 $(\mathrm{C}=\mathrm{O})$. GC-MS $281 \mathrm{~m} / \mathrm{z}[\mathrm{M}-\mathrm{H}]^{+}$(Theory: $280 \mathrm{~m} / \mathrm{z}[\mathrm{M}]^{+}$). Elemental Analysis: C 60.07\%, H 5.80\% (Theory: C 59.99\%, H 5.75\%).

\section{Synthesis of succinic acid mono-(2-phenyl-[1,3]dioxan-5-yl)ester anhydride (2) - $2-($ cis-1,3-O-} benzylidine glycerol)succinic acid monoester $(57.0 \mathrm{~g}, 204.1 \mathrm{mmol})$ was dissolved in DCM (300 $\mathrm{mL})$ in the presence of DCC (25.46 g, $122.5 \mathrm{mmol})$ and stirred for 14 hours. The DCU precipitate was filtered and washed with DCM $(50 \mathrm{~mL})$. The organic phase was added directly to hexanes $(1300 \mathrm{~mL})$. The product was collected by filtration (64\% yield). ${ }^{1} \mathrm{H} \mathrm{NMR}\left(\mathrm{CDCl}_{3}, 400 \mathrm{MHz}\right): \delta 2.75\left(\mathrm{~m}, 4,-\mathrm{CH}_{2}-\mathrm{CH}_{2}-\right), 4.12(\mathrm{~m}$, 4, - $\left.\mathrm{CH}_{2}-\mathrm{CH}-\mathrm{CH}_{2}-\right), 4.25$ (m, 4, - $\left.\mathrm{CH}_{2}-\mathrm{CH}_{-}-\mathrm{CH}_{2}-\right), 4.71$ (m, 2, - $\left.\mathrm{CH}_{2}-\mathrm{CH}-\mathrm{CH}_{2}-\right), 5.52$ (s, 2, CH), 7.34 (m, 3, aromatic $\mathrm{CH}), 7.46(\mathrm{~m}, 2$, aromatic $\mathrm{CH}) .{ }^{13} \mathrm{C} \mathrm{NMR}\left(\mathrm{CDCl}_{3}, 100 \mathrm{MHz}\right): \delta 171.77$ (COOR), $167.99(-$ COOCO-), $137.96(\mathrm{C}), 129.29(\underline{\mathrm{CH}}), 128.51(\underline{\mathrm{CH}}), 126.20(\underline{\mathrm{CH}}), 101.36(\underline{\mathrm{CH}}), 69.13\left(\underline{\mathrm{CH}_{2}}\right), 66.76(\underline{\mathrm{C}} \mathrm{H})$, $30.37\left(\underline{\mathrm{CH}}_{2}\right), 28.94\left(\underline{\mathrm{CH}}_{2}\right)$. FTIR: $v\left(\mathrm{~cm}^{-1}\right) 2938$ (aliph. C-H stretch), $1815(\mathrm{C}=\mathrm{O}), 1730(\mathrm{C}=\mathrm{O})$. FAB- 
MS: $543.2 \mathrm{~m} / \mathrm{z}[\mathrm{M}-\mathrm{H}]^{+}$(Theory: $542.52 \mathrm{~m} / \mathrm{z}[\mathrm{M}]^{+}$). Elemental Analysis: C 61.83\%, H 5.70\% (Theory: C $61.99 \%, \mathrm{H} 5.57 \%)$.

Synthesis of ([G0]-PGLSA-bzld) , $_{\mathbf{2}} \mathbf{P E G}_{\mathbf{2 0 0 0}}(\mathbf{3 c})-\mathrm{PEG}, \mathbf{M}_{\mathrm{w}}=20000(19.34 \mathrm{~g}, 0.967 \mathrm{mmol})$ was dried under vacuum for three hours at $120^{\circ} \mathrm{C}$. The dried PEG was then dissolved in DCM (100 mL) along with 8 eq of succinic acid mono-(2-phenyl-[1,3]dioxan-5-yl)ester anhydride (4.20 g, 7.736 mmol), followed by the addition of $1.6 \mathrm{eq}$ of DMAP $(0.189 \mathrm{~g}, 1.55 \mathrm{mmol})$. The reaction mixture was stirred for one week. Any remaining anhydride was quenched with n-butanol $(1 \mathrm{~mL})$ and allowed to stir for another five hours. The resulting solution was washed with $0.1 \mathrm{~N} \mathrm{HCl}(200 \mathrm{~mL})$, saturated sodium bicarbonate (3 x $200 \mathrm{~mL})$ and brine $(200 \mathrm{~mL})$. The organic phase was dried with sodium sulfate before the solution was concentrated under reduced pressure. A white powder was collected by filtration after precipitation in cold $\left(-20{ }^{\circ} \mathrm{C}\right)$ ethyl ether $\left(96 \%\right.$ yield). ${ }^{1} \mathrm{H}$ NMR $\left(\mathrm{CDCl}_{3}, 400 \mathrm{MHz}\right): \delta 2.69$ (broad m, 4, $\left.-\mathrm{C}_{2}-\mathrm{C}_{2}-\right), 2.73$

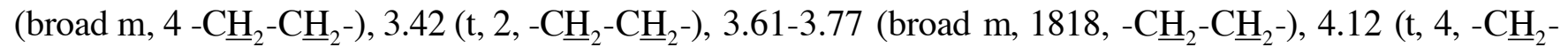

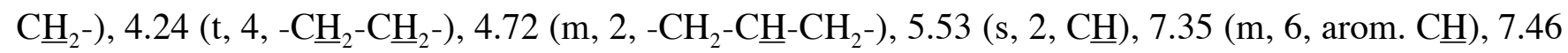
(m, 4, arom. $\underline{\mathrm{CH}}) .{ }^{13} \mathrm{C} \mathrm{NMR}\left(\mathrm{CDCl}_{3}, 100 \mathrm{MHz}\right): \delta 171.68$ (ㅁOR), $137.52(\underline{\mathrm{C}}), 128.61$ (ㅁH), 127.83 $(\underline{\mathrm{CH}}), 125.71(\underline{\mathrm{CH}}), 100.70(\underline{\mathrm{CH}}), 70.23\left(\underline{\mathrm{CH}}_{2}\right), 69.88\left(\underline{\mathrm{CH}}_{2}\right), 69.26\left(\underline{\mathrm{CH}}_{2}\right), 69.04\left(\underline{\mathrm{CH}}_{2}\right), 65.97(\underline{\mathrm{C}} \mathrm{H})$, $63.49\left(\underline{\mathrm{CH}}_{2}\right), 28.92\left(\underline{\mathrm{CH}}_{2}\right)$. MALDI-TOF Mw: 22724, Mn: 22672. SEC Mw: 33489, Mn: 32755, PDI: 1.12. $\mathrm{T}_{\mathrm{m}}=57.9$.

Synthesis of ([G0]-PGLSA-OH) $)_{2}-$ PEG $_{2000}$ (4c)-([G0]-PGLSA-bzld) $)_{2}-$ PEG $_{2000}(15.89$ g, 0.70 mmol) was dissolved in THF (100 mL) before adding $10 \%$ wt Pd/C. The solution was shaken under $\mathrm{H}_{2}$ at 60 psi overnight. The product was filtered over celite and the concentrated solution was precipitated in cold ethyl ether $(1 \mathrm{~L})$ to afford a white powder $94 \%$ yield). ${ }^{1} \mathrm{H} \mathrm{NMR}\left(\mathrm{CDCl}_{3}, 400 \mathrm{MHz}\right): \delta 2.60$ (broad m, 8, -

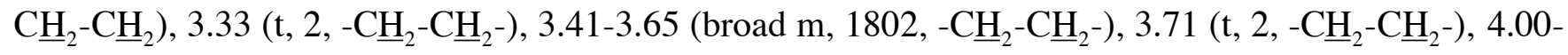
$4.11\left(\mathrm{~m}, 8,-\underline{\mathrm{CH}}_{2}-\mathrm{C}_{2}-\right), 4.80\left(\mathrm{~m}, 2,-\mathrm{CH}_{2}-\mathrm{C}^{\mathrm{H}}-\mathrm{CH}_{2}\right) .{ }^{13} \mathrm{C} \mathrm{NMR}\left(\mathrm{CDCl}_{3}, 100 \mathrm{MHz}\right): \delta 172.24$ (ㅁOR), $70.52\left(\underline{\mathrm{CH}}_{2}\right), 69.73\left(\underline{\mathrm{CH}}_{2}\right), 69.42\left(\underline{\mathrm{CH}}_{2}\right), 68.82\left(\underline{\mathrm{C}} \mathrm{H}_{2}\right), 65.46(\underline{\mathrm{C}} \mathrm{H}), 63.79\left(\underline{\mathrm{CH}}_{2}\right), 63.17\left(\underline{\mathrm{C}} \mathrm{H}_{2}\right), 29.19$ 
$\left(\underline{\mathrm{CH}}_{2}\right), 28.88\left(\underline{\mathrm{CH}}_{2}\right)$. MALDI-TOF Mw: 22512, Mn: 22467. SEC Mw: 22180, Mn: 20467, PDI: 1.13. T $=56.8$.

Synthesis of ([G1]-PGLSA-bzld $)_{\mathbf{2}}-\mathbf{P E G}_{\mathbf{2 0 0 0}}(\mathbf{5 c})-\left([\mathbf{G 0 ] - P G L S A - O H})_{\mathbf{2}}-\mathbf{P E G}_{\mathbf{2 0 0 0 0}}(12.44 \mathrm{~g}, 0.553\right.$ mmol) and 16 eq of succinic acid mono-(2-phenyl-[1,3]dioxan-5-yl)ester anhydride (4.81 g, $8.85 \mathrm{mmol})$ were dissolved in DCM $(75 \mathrm{~mL})$, followed by the addition of $3.2 \mathrm{eq}$ of DMAP $(0.216 \mathrm{~g}, 1.77 \mathrm{mmol})$. The reaction was stirred for one week. Any remaining anhydride was quenched with $\mathrm{n}$-butanol $(1 \mathrm{~mL})$ and allowed to stir for another five hours. The resulting solution was washed with $0.1 \mathrm{~N} \mathrm{HCl}(150 \mathrm{~mL})$, saturated sodium bicarbonate $(3 \times 150 \mathrm{~mL})$ and brine $(150 \mathrm{~mL})$. The organic phase was dried with sodium sulfate before the solution was concentrated under reduced pressure. A white powder was collected by filtration after precipitation in cold $\left(-20^{\circ} \mathrm{C}\right)$ ethyl ether (91\% yield). ${ }^{1} \mathrm{H} \mathrm{NMR}\left(\mathrm{CDCl}_{3}, 400 \mathrm{MHz}\right): \delta 2.61$ (broad m, 16, - $\underline{\mathrm{CH}}_{2}-\mathrm{C}_{2}{ }^{-}$), 2.73 (broad m, 8, - $\mathrm{C}_{\underline{H}_{2}}-\underline{\mathrm{C}}_{2}-$ ), 3.43 (t, 2, - $\mathrm{C}_{2}{ }_{2}-\underline{\mathrm{C}}_{2}-$ ), 3.62-3.69 (broad m,

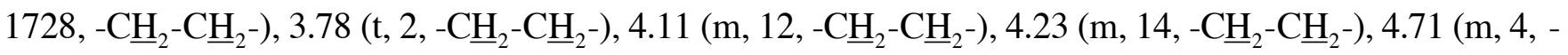
$\left.\mathrm{CH}_{2}-\mathrm{C} \underline{\mathrm{H}}-\mathrm{CH}_{2}-\right), 5.28$ (m, 2, $\left.-\mathrm{CH}_{2}-\mathrm{C}_{\mathbf{H}}-\mathrm{CH}_{2}-\right), 5.52$ (s, 4, $\underline{\mathrm{H}}$ ), 7.33 (m, 12, arom. $\left.\mathrm{C} \underline{\mathrm{H}}\right), 7.46$ (m, 8, arom.

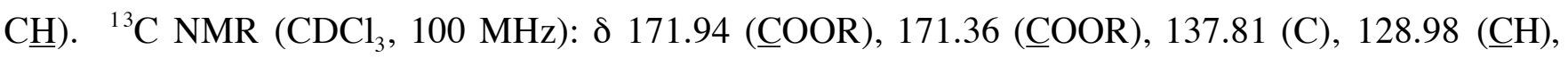
$\left.128.18(\underline{\mathrm{CH}}), 125.97(\underline{\mathrm{CH}}), 101.09(\underline{\mathrm{CH}}), 70.57\left(\underline{\mathrm{CH}}_{2}\right), 70.25(\underline{\mathrm{CH}})_{2}\right), 69.94\left(\underline{\mathrm{CH}}_{2}\right), 66.32(\underline{\mathrm{C}} \mathrm{H}), 63.83$ $\left(\underline{\mathrm{CH}}_{2}\right), 62.28\left(\underline{\mathrm{CH}}_{2}\right), 29.20\left(\underline{\mathrm{CH}}_{2}\right), 28.88\left(\underline{\mathrm{CH}}_{2}\right)$. MALDI-TOF Mw: 23608, Mn: 23564. SEC Mw: 15288, Mn: 14997, PDI: $1.12 . \mathrm{T}_{\mathrm{m}}=56.3$.

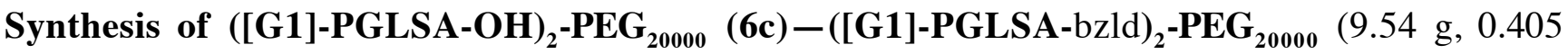
mmol) was dissolved in THF (100 mL) before adding $10 \% \mathrm{wt} \mathrm{Pd} / \mathrm{C}$. The solution was shaken under $\mathrm{H}_{2}$ at 60 psi overnight. The product was filtered over celite and the concentrated solution was precipitated in cold ethyl ether $(1 \mathrm{~L})$ to afford a white powder $\left(91 \%\right.$ yield). ${ }^{1} \mathrm{H} \mathrm{NMR}\left(\mathrm{CDCl}_{3}, 400 \mathrm{MHz}\right)$ : $\delta 2.66$ (broad m, 24,

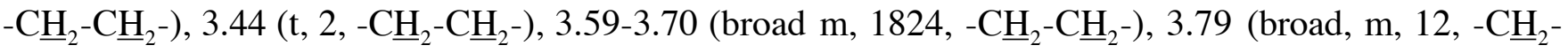
$\left.\mathrm{C}_{2_{2}}\right)$, 4.17-4.28 (m, 14, - $\left.\underline{\mathrm{C}}_{2}-\mathrm{C}_{2}{ }^{-}\right), 4.91$ (m, 4, $\left.-\mathrm{CH}_{2}-\mathrm{C} \underline{H}-\mathrm{CH}_{2}-\right), 5.26\left(\mathrm{~m}, 2,-\mathrm{CH}_{2}-\mathrm{C}_{1}-\mathrm{CH}_{2^{-}}\right) .{ }^{13} \mathrm{C}$ NMR $\left(\mathrm{CDCl}_{3}, 100 \mathrm{MHz}\right): \delta 172.01$ (ㅁO), $70.55\left(\underline{\mathrm{CH}}_{2}\right), 69.94\left(\underline{\mathrm{CH}}_{2}\right), 69.24\left(\underline{\mathrm{CH}}_{2}\right), 68.99(\underline{\mathrm{C}} \mathrm{H}), 63.89$ 
$\left(\underline{\mathrm{CH}}_{2}\right), 62.32\left(\underline{\mathrm{CH}}_{2}\right), 61.78\left(\underline{\mathrm{CH}}_{2}\right), 29.13\left(\underline{\mathrm{CH}}_{2}\right), 28.99\left(\underline{\mathrm{CH}}_{2}\right)$. MALDI-TOF Mw: 23038, Mn: 22999. SEC Mw: 21734, Mn: 20645, PDI: 1.22. $\mathrm{T}_{\mathrm{m}}: 57.2$.

\section{Synthesis of ([G2]-PGLSA-bzld $)_{\mathbf{2}}-\mathbf{P E G}_{\mathbf{2 0 0 0}}(\mathbf{7 c})-([\mathbf{G 1}]-\mathbf{P G L S A - O H})_{\mathbf{2}}-\mathbf{P E G}_{\mathbf{2 0 0 0 0}}(6.98 \mathrm{~g}, 0.301$} $\mathrm{mmol}$ ) and $32 \mathrm{eq}$ of succinic acid mono-(2-phenyl-[1,3]dioxan-5-yl)ester anhydride (5.226 g, 9.63 mmol)) were dissolved in DCM (100 mL), followed by the addition of 6.4 eq of DMAP (0.235 g, $1.93 \mathrm{mmol})$. The reaction was stirred for one week. Any remaining anhydride was quenched with $\mathrm{n}$-butanol (1 mL) and allowed to stir for another five hours. The resulting solution was washed with $0.1 \mathrm{~N} \mathrm{HCl}(150 \mathrm{~mL})$, saturated sodium bicarbonate $(3 \times 150 \mathrm{~mL})$ and brine $(150 \mathrm{~mL})$. The organic phase was dried with sodium sulfate before the solution was concentrated under reduced pressure. A white powder was collected by filtration after precipitation in cold $\left(-20^{\circ} \mathrm{C}\right)$ ethyl ether (86\% yield). ${ }^{1} \mathrm{H} \mathrm{NMR}\left(\mathrm{CDCl}_{3}, 400 \mathrm{MHz}\right): \delta 2.60$ (broad m, 40, - $\underline{\mathrm{CH}}_{2}-\mathrm{C}_{2}{ }^{-}$), 2.72 (broad m, $18-\mathrm{C}_{2}-\underline{\mathrm{CH}}_{2}$ ), 3.44 (t, 2, - $\mathrm{C}_{2}{ }_{2}-\underline{\mathrm{C}}_{2}-$ ), 3.62-3.66 (broad m, 1728, $\left.-\mathrm{CH}_{2}-\mathrm{CH}_{2}-\right), 3.67$ (t, 2, $\left.-\mathrm{CH}_{2}-\underline{\mathrm{CH}}_{2}-\right), 4.12\left(\mathrm{~m}, 28,-\mathrm{CH}_{2}-\mathrm{CH}_{2}-\right), 4.23$ (m, 30, $\left.-\mathrm{CH}_{2}-\mathrm{CH}_{2}-\right), 4.71$ (m, 8, $\mathrm{CH}_{2}-\mathrm{C} \underline{\mathrm{H}}-\mathrm{CH}_{2}-$ ), 5.21 (m, 6, - $\mathrm{CH}_{2}-\mathrm{C}_{\underline{H}}-\mathrm{CH}_{2}-$ ), 5.52 (s, 8, $\left.\mathrm{C} \underline{\mathrm{H}}\right), 7.32$ (m, 24, arom. $\underline{\mathrm{H}}$ ), 7.47 (m, 16, arom.

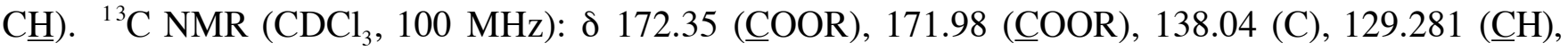
$128.50(\underline{\mathrm{CH}}), 126.23(\underline{\mathrm{CH}}), 101.32(\underline{\mathrm{CH}}), 70.70\left(\underline{\mathrm{CH}}_{2}\right), 70.39\left(\underline{\mathrm{CH}}_{2}\right), 69.10\left(\underline{\mathrm{CH}}_{2}\right), 66.47(\underline{\mathrm{CH}}), 62.41$ $\left(\underline{\mathrm{CH}}_{2}\right), 29.24\left(\underline{\mathrm{CH}}_{2}\right), 28.91\left(\underline{\mathrm{CH}}_{2}\right)$. MALDI-TOF Mw: 26077, Mn: 26031. SEC Mw: 30100, Mn: 28418, PDI: 1.12. $\mathrm{T}_{\mathrm{m}}: 56.1$.

\section{Synthesis of ([G2]-PGLSA-OH) $)_{2}-$ PEG $_{2000}(\mathbf{8 c})-([\mathbf{G 2}]-P G L S A-b z l d)_{2}-$ PEG $_{20000}(5.79$ g, 0.229} mmol) was dissolved in THF (75 mL) before adding $10 \%$ wt $\mathrm{Pd} / \mathrm{C}$. The solution was shaken under $\mathrm{H}_{2}$ at 60 psi overnight. The product was filtered over celite and the concentrated solution was precipitated in cold ethyl ether $(1 \mathrm{~L})$ to afford a white powder $(89 \%$ yield $) .{ }^{1} \mathrm{H} \mathrm{NMR}\left(\mathrm{CDCl}_{3}, 400 \mathrm{MHz}\right): \delta 2.64$ (broad m, 56,

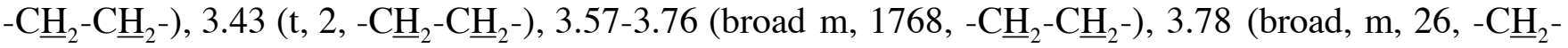
$\left.\mathrm{C}_{2_{2}}^{-}\right), 4.15-4.27$ (m, 32, $\left.-\underline{\mathrm{CH}}_{2}-\mathrm{C}_{2}-\right), 4.90$ (m, 8, $\left.-\mathrm{CH}_{2}-\mathrm{C} \underline{H}-\mathrm{CH}_{2}-\right), 5.25$ (m, 6, - $\left.\mathrm{CH}_{2}-\mathrm{CH}_{-}-\mathrm{CH}_{2}-\right) .{ }^{13} \mathrm{C}$

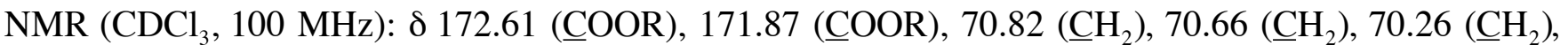
$69.98\left(\underline{\mathrm{CH}}_{2}\right), 69.12\left(\underline{\mathrm{CH}}_{2}\right), 65.79(\underline{\mathrm{CH}}), 64.08\left(\underline{\mathrm{CH}}_{2}\right), 63.42\left(\underline{\mathrm{CH}}_{2}\right), 62.47\left(\underline{\mathrm{CH}}_{2}\right), 61.86\left(\underline{\mathrm{CH}}_{2}\right), 29.18$ 
$\left(\underline{\mathrm{CH}}_{2}\right), 28.89\left(\underline{\mathrm{CH}}_{2}\right), 28.80\left(\underline{\mathrm{CH}}_{2}\right)$. MALDI-TOF Mw: 25491, Mn: 25455. SEC Mw: 21235, Mn: 19924, PDI: $1.17 . \mathrm{T}_{\mathrm{m}}: 53.9$.

\section{Synthesis of ([G3]-PGLSA-bzld $)_{2}-$ PEG $_{2000}(\text { 9c) - ([G2]-PGLSA-OH })_{2}-$ PEG $_{2000}(4.32$ g, 0.176} mmol) and succinic acid 64 eq of mono-(2-phenyl-[1,3]dioxan-5-yl)ester anhydride (6.102 g, 11.25 mmol) were dissolved in DCM (100 mL), followed by the addition of 12.8 eq DMAP $(0.275 \mathrm{~g}, 2.25 \mathrm{mmol})$. The reaction was stirred for one week. Any remaining anhydride was quenched with $\mathrm{n}$-butanol $(1 \mathrm{~mL})$ and allowed to stir for another five hours. The resulting solution was washed with $0.1 \mathrm{~N} \mathrm{HCl}(150 \mathrm{~mL})$, saturated sodium bicarbonate $(3 \times 150 \mathrm{~mL})$ and brine $(150 \mathrm{~mL})$. The organic phase was dried with sodium sulfate before the solution was concentrated under reduced pressure. A white powder was collected by filtration after precipitation in cold $\left(-20{ }^{\circ} \mathrm{C}\right)$ ethyl ether $\left(92 \%\right.$ yield). ${ }^{1} \mathrm{H} \mathrm{NMR}\left(\mathrm{CDCl}_{3}, 400 \mathrm{MHz}\right): \delta 2.59$ (broad m, 80, - $\underline{\mathrm{CH}}_{2}-\mathrm{C}_{2}{ }^{-}$), 2.71 (broad m, $42-\underline{\mathrm{CH}}_{2}-\underline{\mathrm{C}}_{2}-$ ), 3.44 (t, 2, - $\mathrm{C}_{2}{ }_{2}-\underline{\mathrm{C}}_{2}-$ ), 3.60-3.66 (broad m, 1790, $\left.-\mathrm{CH}_{2}-\mathrm{CH}_{2}-\right), 3.71\left(\mathrm{t}, 2,-\mathrm{CH}_{2}-\underline{\mathrm{CH}}_{2}-\right), 4.11\left(\mathrm{~m}, 63,-\mathrm{CH}_{2}-\mathrm{CH}_{2}-\right), 4.22\left(\mathrm{~m}, 63,-\underline{\mathrm{C}}_{2}-\mathrm{CH}_{2}-\right), 4.70$ (m, 16, $\left.-\mathrm{CH}_{2}-\mathrm{C} \underline{\mathrm{H}}-\mathrm{CH}_{2}-\right), 5.21$ (m, 14, $\left.-\mathrm{CH}_{2}-\mathrm{C}_{\underline{H}}-\mathrm{CH}_{2}-\right), 5.51$ (s, 16, $\left.\mathrm{C} \underline{\mathrm{H}}\right), 7.33$ (m, 46, arom. $\left.\mathrm{C} \underline{\mathrm{H}}\right), 7.46$ (m, 29, arom. $\underline{\mathrm{CH}}) .{ }^{13} \mathrm{C} \mathrm{NMR}\left(\mathrm{CDCl}_{3}, 100 \mathrm{MHz}\right): \delta 172.39$ (ㅁOOR), 138.47 (C), $129.68(\underline{\mathrm{C}} \mathrm{H}), 128.90(\underline{\mathrm{C}} \mathrm{H})$, $126.64(\underline{\mathrm{CH}}), 101.70(\underline{\mathrm{C}} \mathrm{H}), 71.12\left(\underline{\mathrm{CH}}_{2}\right), 69.84\left(\underline{\mathrm{CH}}_{2}\right), 69.50\left(\underline{\mathrm{CH}}_{2}\right), 66.87(\underline{\mathrm{CH}}), 64.41\left(\underline{\mathrm{CH}}_{2}\right), 62.80$ $\left(\underline{\mathrm{CH}}_{2}\right), 29.64\left(\underline{\mathrm{CH}}_{2}\right), 29.12\left(\underline{\mathrm{CH}}_{2}\right)$. MALDI-TOF Mw: 29521, Mn: 29481. SEC Mw: 14885, Mn: 14474, PDI: $1.13 . \mathrm{T}_{\mathrm{m}}: 55.3$.

\section{Synthesis of ([G3]-PGLSA-OH $)_{2}-$ PEG $_{20000}(\text { (10c) - ([G3]-PGLSA-bzld })_{2}-$ PEG $_{20000}(3.57$ g, 0.124}

mmol) was dissolved in THF (30 mL) before adding $\mathrm{Pd} / \mathrm{C}$. The solution was shaken under $\mathrm{H}_{2}$ at 60 psi overnight. The product was filtered over celite and the concentrated solution was precipitated in cold ethyl ether $(1 \mathrm{~L})$ to afford a white powder $\left(87 \%\right.$ yield). ${ }^{1} \mathrm{H} \mathrm{NMR}\left(\mathrm{CDCl}_{3}, 400 \mathrm{MHz}\right)$ : $\delta 2.64$ (broad m, 124, $\mathrm{CH}_{2}-\underline{\mathrm{CH}}_{2}-$ ), 3.42 (t, 2, $-\mathrm{C}_{2}-\underline{\mathrm{CH}}_{2}-$ ), 3.57-3.71 (broad m, 1793, - $\mathrm{C}_{2}-\mathrm{C}_{2}{ }^{-}$), 3.80 (broad, m, 60, - $\mathrm{C}_{2^{-}}$ $\left.\mathrm{C}_{2}\right), 4.19\left(\mathrm{~m}, 40,-\underline{\mathrm{CH}}_{2}-\mathrm{C}_{2}{ }^{-}\right), 4.26\left(\mathrm{~m}, 30,-\underline{\mathrm{C}}_{2}-\mathrm{C}_{2}-\right), 4.89$ (m, 16, - $\left.\mathrm{CH}_{2}-\mathrm{C}_{-}-\mathrm{CH}_{2}-\right), 5.24$ (m, 14, $\left.\mathrm{CH}_{2}-\mathrm{C} \underline{\mathrm{H}}-\mathrm{CH}_{2}-\right) .{ }^{13} \mathrm{C} \mathrm{NMR}\left(\mathrm{CDCl}_{3}, 100 \mathrm{MHz}\right): \delta 172.44(\underline{\mathrm{COOR}}), 71.48\left(\underline{\mathrm{CH}_{2}}\right), 70.64\left(\underline{\mathrm{CH}_{2}}\right), 69.48$ 
$\left(\underline{\mathrm{CH}}_{2}\right), 65.87(\underline{\mathrm{CH}}), 62.50\left(\underline{\mathrm{CH}}_{2}\right), 61.35\left(\underline{\mathrm{CH}}_{2}\right), 29.14\left(\underline{\mathrm{CH}}_{2}\right)$. MALDI-TOF Mw: 27987, Mn: 27958. SEC Mw: 26644, Mn: 25546, PDI: 1.22. $\mathrm{T}_{\mathrm{m}}: 52.3$.

\section{Synthesis of ([G4]-PGLSA-bzld $)_{2}-$ PEG $_{\mathbf{2 0 0 0}}\left(\right.$ 11c) $-([G 3]-P G L S A-O H)_{2}-$ PEG $_{2000}(2.57$ g, 0.0939}

mmol) and 128 eq of succinic acid mono-(2-phenyl-[1,3]dioxan-5-yl)ester anhydride (6.521 g, 12.02 mmol) were dissolved in DCM (100 mL), followed by the addition of 25.6 eq of DMAP (0.294 g, 2.403 mmol). The reaction was stirred for one week. Any remaining anhydride was quenched with n-butanol (1 $\mathrm{mL})$ and allowed to stir for another five hours. The resulting solution was washed with $0.1 \mathrm{~N} \mathrm{HCl}(150$ $\mathrm{mL})$, saturated sodium bicarbonate $(3 \times 150 \mathrm{~mL})$ and brine $(150 \mathrm{~mL})$. The organic phase was dried with sodium sulfate before the solution was concentrated under reduced pressure. A white powder was collected by filtration after precipitation in cold $\left(-20^{\circ} \mathrm{C}\right)$ ethyl ether $(87 \%$ yield $) .{ }^{1} \mathrm{H}$ NMR $\left(\mathrm{CDCl}_{3}, 400\right.$ $\mathrm{MHz}): \delta 2.60$ (broad m, 184, $-\mathrm{C}_{2}-\underline{\mathrm{CH}}_{2}-$ ), 2.71 (broad m, $56-\mathrm{C}_{2}-\underline{\mathrm{CH}}_{2}-$ ), 3.43 (t, 2, $-\mathrm{C}_{2}-\mathrm{C}_{2}-$ ), 3.58-

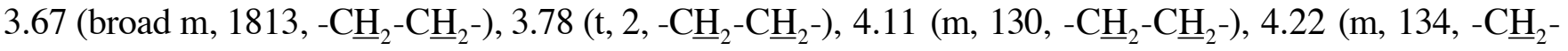
$\left.\mathrm{C}_{2_{2}}^{-}\right), 4.70$ (m, 32, - $\left.\mathrm{CH}_{2}-\mathrm{C}_{\mathbf{H}}-\mathrm{CH}_{2}-\right), 5.22$ (m, 30, - $\left.\mathrm{CH}_{2}-\mathrm{C} \underline{\mathrm{H}}-\mathrm{CH}_{2}-\right), 5.51$ (s, 32, $\underline{\mathrm{H}}$ ), 7.32 (m, 90, arom.

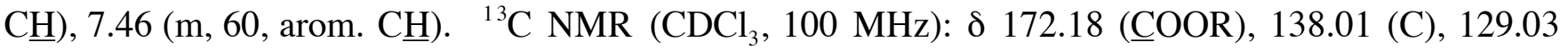
$(\underline{\mathrm{CH}}), 128.28(\underline{\mathrm{CH}}), 126.11(\underline{\mathrm{CH}}), 101.07(\underline{\mathrm{CH}}), 71.76\left(\underline{\mathrm{CH}}_{2}\right), 70.46(\underline{\mathrm{CH}}), 69.10\left(\underline{\mathrm{CH}}_{2}\right), 66.43(\underline{\mathrm{CH}}), 62.50$ $\left(\underline{\mathrm{CH}}_{2}\right), 29.24\left(\underline{\mathrm{CH}}_{2}\right), 29.18\left(\underline{\mathrm{CH}}_{2}\right), 28.80\left(\underline{\mathrm{CH}}_{2}\right)$. MALDI-TOF Mw: 36257, Mn: 36029. SEC Mw: 11877, Mn: 8528, PDI: 1.66. $\mathrm{T}_{\mathrm{m}}: 45.3$.

\section{Synthesis of ([G4]-PGLSA-OH) $)_{2}-$ PEG $_{20000}(12 c)-([G 4]-P G L S A-b z l d)_{2}-$ PEG $_{20000}(1.97$ g, 0.0551} mmol) was dissolved in THF (20 mL) before adding $10 \%$ wt $\mathrm{Pd} / \mathrm{C}$. The solution was shaken under $\mathrm{H}_{2}$ at 60 psi overnight. The product was filtered over celite and the concentrated solution was precipitated in cold ethyl ether $(1 \mathrm{~L})$ to afford a white powder (90\% yield). ${ }^{1} \mathrm{H} \mathrm{NMR}\left(\mathrm{CDCl}_{3}, 400 \mathrm{MHz}\right): \delta 2.65$ (broad m, 230, $-\mathrm{C}_{2}-\mathrm{CH}_{2}-$ ), 3.45 (t, 2, $-\mathrm{C}_{2}-\mathrm{CH}_{2}-$ ), 3.57-3.67 (broad m, 1767, $-\mathrm{C}_{2}-\mathrm{C}_{2}{ }^{-}$), 3.80 (broad, m, 60, $\left.\mathrm{C}_{\mathrm{H}_{2}}-\underline{\mathrm{C}}_{2}-\right), 4.20\left(\mathrm{~m}, 80,-\underline{\mathrm{CH}}_{2}-\mathrm{C}_{2}-\right), 4.28\left(\mathrm{~m}, 83,-\underline{\mathrm{CH}}_{2}-\mathrm{C}_{2}-\right), 4.88\left(\mathrm{~m}, 32,-\mathrm{CH}_{2}-\mathrm{C} \underline{\mathrm{H}}-\mathrm{CH}_{2}-\right), 5.25$ (m, 30 , $\left.-\mathrm{CH}_{2}-\mathrm{C} \underline{\mathrm{H}}-\mathrm{CH}_{2}-\right) .{ }^{13} \mathrm{C} \mathrm{NMR}\left(\mathrm{CDCl}_{3}, 100 \mathrm{MHz}\right): \delta 172.98$ (ㅁOOR), $70.66\left(\underline{\mathrm{CH}_{2}}\right), 70.06\left(\underline{\mathrm{CH}_{2}}\right), 65.73$ 
$(\underline{\mathrm{CH}}), 63.32\left(\underline{\mathrm{CH}}_{2}\right), 61.09\left(\underline{\mathrm{CH}}_{2}\right), 28.95\left(\underline{\mathrm{CH}}_{2}\right)$. MALDI-TOF N/A. SEC Mw: 18997, Mn: 13920, PDI: 1.29. $\mathrm{T}_{\mathrm{m}}: 49.2$.

Synthesis of ([G0]-PGLSA-bzld) $)_{\mathbf{2}}-\mathbf{P E G}_{\mathbf{1 0 0 0}} \mathbf{( 3 b )}$ - This was prepared from dry PEG, Mw = 10000, (19.34 g, $1.934 \mathrm{mmol})$ with the addition of 8 eq of succinic acid mono-(2-phenyl-[1,3]dioxan-5-yl)ester anhydride (8.39 g, $15.47 \mathrm{mmol})$ and $1.6 \mathrm{eq}$ of DMAP (0.378 g, $3.094 \mathrm{mmol})$ while stirring for three days in DCM (100 mL). Any remaining anhydride was quenched with n-butanol $(1 \mathrm{~mL})$ and allowed to stir for another five hours. The resulting solution was washed with $0.1 \mathrm{~N} \mathrm{HCl}(200 \mathrm{~mL})$, saturated sodium bicarbonate $(3 \times 200 \mathrm{~mL})$ and brine $(200 \mathrm{~mL})$. The organic phase was dried with sodium sulfate before the solution was concentrated under reduced pressure. A white powder was collected by filtration after precipitation in cold $\left(-20^{\circ} \mathrm{C}\right)$ ethyl ether $\left(96 \%\right.$ yield). ${ }^{1} \mathrm{H} \mathrm{NMR}\left(\mathrm{CDCl}_{3}, 400 \mathrm{MHz}\right): \delta 2.68$ (broad m, 4, $\mathrm{C}_{\underline{H}_{2}}-\underline{\mathrm{C}}_{2}-$ ), 2.73 (broad m, $4-\mathrm{C}_{2}-\mathrm{C}_{2}-$ ), 3.36 (t, 2, $-\mathrm{C}_{2}-\mathrm{C}_{2}-$ ), 3.49-3.70 (broad m, 904, - $\underline{\mathrm{H}}_{2}-\mathrm{C}_{2}-$ ),

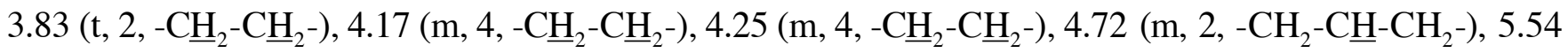
(m, 2, $\left.-\mathrm{CH}_{2}-\mathrm{C}_{\mathrm{H}}-\mathrm{CH}_{2}-\right), 7.34$ (m, 6, arom. $\left.\mathrm{C} \underline{\mathrm{H}}\right), 7.47$ (m, 4, arom. $\left.\mathrm{C} \underline{\mathrm{H}}\right) .{ }^{13} \mathrm{C} \mathrm{NMR}\left(\mathrm{CDCl}_{3}, 100 \mathrm{MHz}\right)$ :

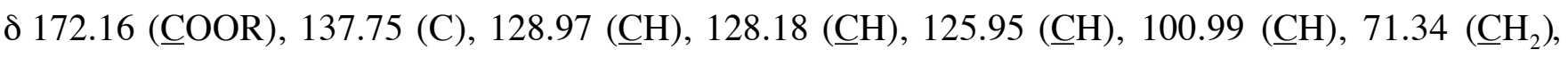
$70.25\left(\underline{\mathrm{CH}}_{2}\right), 70.09\left(\underline{\mathrm{CH}}_{2}\right), 69.46\left(\underline{\mathrm{CH}}_{2}\right), 68.84\left(\underline{\mathrm{CH}}_{2}\right), 66.06(\underline{\mathrm{CH}}), 63.71\left(\underline{\mathrm{CH}}_{2}\right), 29.05\left(\underline{\mathrm{CH}}_{2}\right), 28.79$ $\left(\underline{\mathrm{CH}}_{2}\right)$. MALDI-TOF Mw: 13487, Mn: 13427. SEC Mw: 12910, Mn: 12016, PDI: 1.07. T $\mathrm{T}_{\mathrm{m}}: 54.7$.

\section{Synthesis of ([G0]-PGLSA-OH $)_{\mathbf{2}}$-PEG $_{\mathbf{1 0 0 0}}(\mathbf{4 b})-\left([\text { G0]-PGLSA-bzld })_{\mathbf{2}}-\right.$ PEG $_{1000}(17.23$ g, 1.278}

mmol) was dissolved in THF $(150 \mathrm{~mL})$ before adding $10 \% \mathrm{wt} \mathrm{Pd} / \mathrm{C}$. The solution was shaken under $\mathrm{H}_{2}$ at 60 psi overnight. The product was filtered over celite and the concentrated solution was precipitated in cold ethyl ether $(1 \mathrm{~L})$ to afford a white powder $\left(95 \%\right.$ yield). ${ }^{1} \mathrm{H} \mathrm{NMR}\left(\mathrm{CDCl}_{3}, 400 \mathrm{MHz}\right)$ : $\delta 2.64$ (broad m, 8, $\mathrm{C}_{\mathrm{H}_{2}}-\underline{\mathrm{C}}_{2}-$ ), 3.42 (t, 2, - $\underline{\mathrm{CH}}_{2}-\underline{\mathrm{CH}}_{2}-$ ), 3.56-3.68 (broad m, 984, - $\underline{\mathrm{CH}}_{2}-\mathrm{C}_{2}-$ ), 3.77 (broad, m, 4, - $\underline{\mathrm{H}}_{2}-\mathrm{C}_{2}-$ ), $4.21\left(\mathrm{~m}, 4,-\mathrm{CH}_{2}-\mathrm{CH}_{2}-\right), 4.87\left(\mathrm{~m}, 2,-\mathrm{CH}_{2}-\mathrm{CH}-\mathrm{CH}_{2}-\right) .{ }^{13} \mathrm{C} \mathrm{NMR}\left(\mathrm{CDCl}_{3}, 100 \mathrm{MHz}\right): \delta 172.41$ (COOR), $71.44\left(\underline{\mathrm{CH}}_{2}\right), 70.50\left(\underline{\mathrm{CH}}_{2}\right), 69.33\left(\underline{\mathrm{CH}}_{2}\right), 68.70\left(\underline{\mathrm{CH}}_{2}\right), 65.37(\underline{\mathrm{CH}}), 63.50\left(\underline{\mathrm{CH}}_{2}\right), 62.43\left(\underline{\mathrm{CH}}_{2}\right), 61.79$ 
$\left(\underline{\mathrm{CH}}_{2}\right), 61.57\left(\underline{\mathrm{CH}}_{2}\right), 29.37\left(\underline{\mathrm{CH}}_{2}\right), 29.21\left(\underline{\mathrm{CH}}_{2}\right)$. MALDI-TOF Mw: 13251, Mn: 13193. SEC Mw: 11922, Mn: 11373, PDI: 1.12. $\mathrm{T}_{\mathrm{m}}: 55.8$.

\section{Synthesis of ([G1]-PGLSA-bzld) $)_{2}-$ PEG $_{1000}(5 \mathbf{b})$ - This was prepared from ([G0]-PGLSA-OH) $)_{2}$ -} PEG $_{10000}(14.65 \mathrm{~g}, 1.11 \mathrm{mmol}), 16$ eq of succinic acid mono-(2-phenyl-[1,3]dioxan-5-yl)ester anhydride $(9.58 \mathrm{~g}, 17.66 \mathrm{mmol})$ and $3.2 \mathrm{eq}$ of DMAP $(0.431 \mathrm{~g}, 3.53 \mathrm{mmol})$ and allowed to stir for three days in DCM (100 mL). Any remaining anhydride was quenched with n-butanol $(1 \mathrm{~mL})$ and allowed to stir for another five hours. The resulting solution was washed with $0.1 \mathrm{~N} \mathrm{HCl}(200 \mathrm{~mL})$, saturated sodium bicarbonate $(3 \times 200 \mathrm{~mL})$ and brine $(200 \mathrm{~mL})$. The organic phase was dried with sodium sulfate before the solution was concentrated under reduced pressure. A white powder was collected by filtration after precipitation in cold $\left(-20^{\circ} \mathrm{C}\right)$ ethyl ether $\left(91 \%\right.$ yield). ${ }^{1} \mathrm{H} \mathrm{NMR}\left(\mathrm{CDCl}_{3}, 400 \mathrm{MHz}\right): \delta 2.64$ (broad m, 16, $\mathrm{C}_{\underline{H}_{2}}-\underline{\mathrm{C}}_{2}-$ ), 2.71 (broad m, $8-\mathrm{C}_{2}-\underline{\mathrm{CH}}_{2}$ ), 3.40 (t, 2, - $\underline{\mathrm{CH}}_{2}-\mathrm{C}_{2}-$ ), 3.59-3.72 (broad m, 924, - $\underline{\mathrm{H}}_{2}-\mathrm{C}_{2}-$ ),

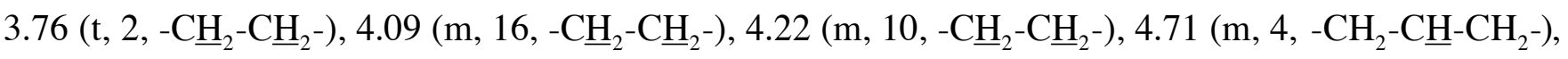
5.26 (m, 2, $\left.-\mathrm{CH}_{2}-\mathrm{C} \underline{\mathrm{H}}-\mathrm{CH}_{2}-\right), 5.53$ (s, 4, $\underline{\mathrm{H}}$ ), 7.30 (m, 12, arom. $\left.\mathrm{C} \underline{\mathrm{H}}\right), 7.44$ (m, 8, arom. $\left.\mathrm{C} \underline{\mathrm{H}}\right) .{ }^{13} \mathrm{C}$ NMR $\left(\mathrm{CDCl}_{3}, 100 \mathrm{MHz}\right): \delta 172.03$ (ㅁO), $137.79(\mathrm{C}), 129.64(\underline{\mathrm{CH}}), 128.98(\underline{\mathrm{C}} \mathrm{H}), 128.20(\underline{\mathrm{C}} \mathrm{H}), 125.95$ (ㅌH), $100.99(\underline{\mathrm{CH}}), 70.42\left(\underline{\mathrm{CH}}_{2}\right), 70.11\left(\underline{\mathrm{CH}}_{2}\right), 68.87\left(\underline{\mathrm{CH}}_{2}\right), 68.80\left(\underline{\mathrm{CH}}_{2}\right), 66.18(\underline{\mathrm{CH}}), 63.72\left(\underline{\mathrm{CH}}_{2}\right), 62.13$ $\left(\underline{\mathrm{CH}}_{2}\right), 28.97\left(\underline{\mathrm{CH}}_{2}\right), 28.65\left(\underline{\mathrm{CH}}_{2}\right)$. MALDI-TOF Mw: 14219, Mn: 14259. SEC Mw: 13422, Mn: 12602, PDI: $1.07 . \mathrm{T}_{\mathrm{m}}: 53.9$.

\section{Synthesis of ([G1]-PGLSA-OH $)_{2}-$ PEG $_{10000}(6 b)-([G 1]-P G L S A-b z l d)_{2}-$ PEG $_{10000}(12.57$ g, 0.878} mmol) was dissolved in THF (125 mL) before adding $10 \%$ wt Pd/C. The solution was shaken under $\mathrm{H}_{2}$ at 60 psi overnight. The product was filtered over celite and the concentrated solution was precipitated in cold ethyl ether $(1 \mathrm{~L})$ to afford a white powder $\left(94 \%\right.$ yield). ${ }^{1} \mathrm{H} \mathrm{NMR}\left(\mathrm{CDCl}_{3}, 400 \mathrm{MHz}\right)$ : $\delta 2.62$ (broad m, 24, $-\mathrm{CH}_{2}-\mathrm{CH}_{2}-$ ), 3.40 (t, 2, $-\underline{\mathrm{CH}}_{2}-\underline{\mathrm{CH}}_{2}-$ ), 3.55-3.69 (broad m, 936, - $\underline{\mathrm{H}}_{2}-\underline{\mathrm{CH}}_{2}-$ ), 3.76 (broad, m, 14, - $\underline{\mathrm{CH}}_{2}-\mathrm{C}_{2}$ ),

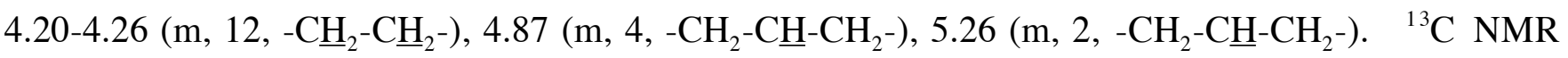

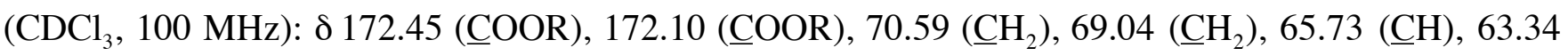


$\left(\underline{\mathrm{CH}}_{2}\right), 62.38\left(\underline{\mathrm{CH}}_{2}\right), 28.96\left(\underline{\mathrm{CH}}_{2}\right), 28.86\left(\underline{\mathrm{CH}}_{2}\right)$. MALDI-TOF Mw: 14013, Mn: 13961. SEC Mw: 11765, Mn: 11064, PDI: 1.06. $\mathrm{T}_{\mathrm{m}}: 53.4$.

\section{Synthesis of ([G2]-PGLSA-bzld $)_{2}-\mathbf{P E G}_{10000}(\mathbf{7 b})$ - This was prepared from ([G1]-PGLSA-OH) $)_{2}$ -}

PEG $_{10000}(9.87 \mathrm{~g}, 0.707 \mathrm{mmol})$ and $32 \mathrm{eq}$ of succinic acid mono-(2-phenyl-[1,3]dioxan-5-yl)ester anhydride (12.26 g, $22.61 \mathrm{mmol})$ and $6.4 \mathrm{eq}$ of DMAP $(0.552 \mathrm{~g}, 4.52 \mathrm{mmol})$. The reaction was stirred for three days in DCM (50 mL). Any remaining anhydride was quenched with n-butanol (1 mL) and allowed to stir for another five hours. The resulting solution was washed with $0.1 \mathrm{~N} \mathrm{HCl}(200 \mathrm{~mL})$, saturated sodium bicarbonate $(3 \times 200 \mathrm{~mL})$ and brine $(200 \mathrm{~mL})$. The organic phase was dried with sodium sulfate before the solution was concentrated under reduced pressure. A white powder was collected by filtration after precipitation in cold $\left(-20^{\circ} \mathrm{C}\right)$ ethyl ether (86\% yield). ${ }^{1} \mathrm{H} \mathrm{NMR}\left(\mathrm{CDCl}_{3}, 400 \mathrm{MHz}\right): \delta 2.58$ (broad m, 40, $-\underline{\mathrm{CH}}_{2}-\underline{\mathrm{CH}}_{2}-$ ), 2.70 (broad m, $18-\underline{\mathrm{CH}}_{2}-\underline{\mathrm{CH}}_{2}-$ ), 3.42 (t, 2, - $\underline{\mathrm{C}}_{2}-\underline{\mathrm{CH}}_{2}-$ ), 3.55-3.61 (broad m, 948, - $\underline{\mathrm{H}}_{2}-$

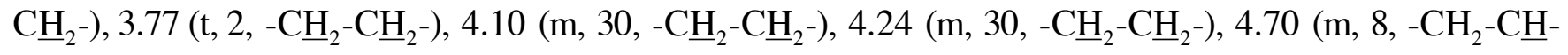
$\left.\mathrm{CH}_{2}{ }^{-}\right), 5.21$ (m, 6, - $\left.\mathrm{CH}_{2}-\mathrm{C}_{\underline{H}}-\mathrm{CH}_{2}-\right), 5.50$ (s, 8, $\underline{\mathrm{H}}$ ), 7.36 (m, 24, arom. $\mathrm{C} \underline{\mathrm{H}}$ ), 7.47 (m, 16, arom. $\left.\mathrm{C} \underline{\mathrm{H}}\right) .{ }^{13} \mathrm{C}$

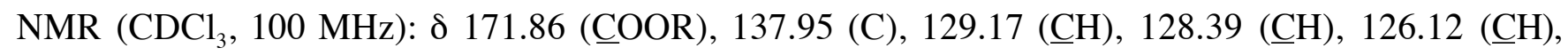
$101.19(\underline{\mathrm{C}} \mathrm{H}), 70.76\left(\underline{\mathrm{CH}}_{2}\right), 69.32\left(\underline{\mathrm{CH}}_{2}\right), 68.97\left(\underline{\mathrm{CH}}_{2}\right), 66.36(\underline{\mathrm{CH}}), 63.90\left(\underline{\mathrm{CH}}_{2}\right), 62.29\left(\underline{\mathrm{CH}}_{2}\right), 29.13$ $\left(\underline{\mathrm{CH}}_{2}\right), 28.80\left(\underline{\mathrm{CH}}_{2}\right), 28.61\left(\underline{\mathrm{CH}}_{2}\right)$. MALDI-TOF Mw: 16048, Mn: 16003. SEC Mw: 14440, Mn: 13515, PDI: 1.07. $\mathrm{T}_{\mathrm{m}}: 52.1$.

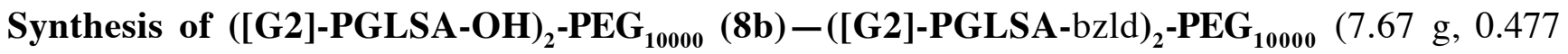
mmol) was dissolved in THF (50 mL) before adding $10 \%$ wt $\mathrm{Pd} / \mathrm{C}$. The solution was shaken under $\mathrm{H}_{2}$ at 60 psi overnight. The product was filtered over celite and the concentrated solution was precipitated in cold ethyl ether $(1 \mathrm{~L})$ to afford a white powder $\left(92 \%\right.$ yield). ${ }^{1} \mathrm{H} \mathrm{NMR}\left(\mathrm{CDCl}_{3}, 400 \mathrm{MHz}\right)$ : $\delta 2.63$ (broad m, 56, $-\mathrm{CH}_{2}-\mathrm{CH}_{2}-$ ), 3.42 (t, 2, $-\underline{\mathrm{CH}}_{2}-\underline{\mathrm{CH}}_{2}-$ ), 3.59-3.78 (broad m, 949, - $\underline{\mathrm{CH}}_{2}-\underline{\mathrm{CH}}_{2}-$ ), 3.80 (broad, m, 30, - $\underline{\mathrm{CH}}_{2}-\mathrm{C}_{2}$ ), 4.16-4.29 (m, 28, - $\left.\mathrm{CH}_{2}-\mathrm{CH}_{2}-\right), 4.89$ (m, 8, - $\left.\mathrm{CH}_{2}-\mathrm{C} \underline{\mathrm{H}}-\mathrm{CH}_{2}-\right), 5.24$ (m, 6, - $\left.\mathrm{CH}_{2}-\mathrm{C}_{\mathbf{H}}-\mathrm{CH}_{2}-\right) .{ }^{13} \mathrm{C} \mathrm{NMR}$

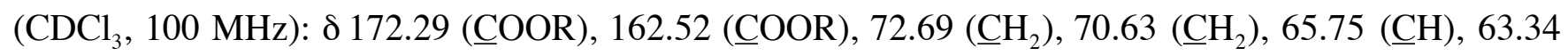


$\left(\underline{\mathrm{CH}}_{2}\right), 62.51\left(\underline{\mathrm{CH}}_{2}\right), 29.01\left(\underline{\mathrm{CH}}_{2}\right)$. MALDI-TOF Mw: 15318, Mn: 15275. SEC Mw: 15132, Mn: 12710, PDI: 1.19. $\mathrm{T}_{\mathrm{m}}: 52.3$.

\section{Synthesis of ([G3]-PGLSA-bzld) $)_{2}-\mathbf{P E G}_{1000}(\mathbf{9 b})$ - This was prepared from ([G2]-PGLSA-OH) $)_{2}$ -}

PEG $_{10000}(4.88 \mathrm{~g}, 0.318 \mathrm{mmol})$ and $64 \mathrm{eq}$ of succinic acid mono-(2-phenyl-[1,3]dioxan-5-yl)ester anhydride (11.03 g, $20.33 \mathrm{mmol})$ and $12.8 \mathrm{eq}$ of DMAP (0.497 g, $4.07 \mathrm{mmol})$. The reaction was stirred for three days in DCM $(50 \mathrm{~mL})$. Any remaining anhydride was quenched with n-butanol $(1 \mathrm{~mL})$ and allowed to stir for another five hours. The resulting solution was washed with $0.1 \mathrm{~N} \mathrm{HCl}(200 \mathrm{~mL})$, saturated sodium bicarbonate $(3 \times 200 \mathrm{~mL})$ and brine $(200 \mathrm{~mL})$. The organic phase was dried with sodium sulfate before the solution was concentrated under reduced pressure. A white powder was collected by filtration after precipitation in cold $\left(-20^{\circ} \mathrm{C}\right)$ ethyl ether (92\% yield). ${ }^{1} \mathrm{H} \mathrm{NMR}\left(\mathrm{CDCl}_{3}, 400 \mathrm{MHz}\right): \delta 2.62$ (broad m, 84, - $\underline{\mathrm{CH}}_{2}-\mathrm{C}_{2}{ }^{-}$), 2.78 (broad m, $42-\underline{\mathrm{C}}_{2}-\underline{\mathrm{C}}_{2}-$ ), 3.47 (t, 2, - $\mathrm{C}_{2}{ }_{2}-\underline{\mathrm{C}}_{2}-$ ), 3.64-3.69 (broad m, 897, $\left.-\mathrm{CH}_{2}-\mathrm{CH}_{2}-\right), 3.81$ (t, 2, $\left.-\mathrm{CH}_{2}-\mathrm{CH}_{2}-\right), 4.16\left(\mathrm{~m}, 60,-\mathrm{CH}_{2}-\mathrm{CH}_{2}-\right), 4.27$ (m, 70, - $\left.\underline{\mathrm{H}}_{2}-\mathrm{CH}_{2}-\right), 4.73$ (m, 16, $\left.\mathrm{CH}_{2}-\mathrm{C} \underline{\mathrm{H}}-\mathrm{CH}_{2}-\right), 5.23$ (m, 14, - $\left.\mathrm{CH}_{2}-\mathrm{C}_{\underline{H}}-\mathrm{CH}_{2}-\right), 5.54$ (s, 16, $\left.\mathrm{C} \underline{\mathrm{H}}\right), 7.34$ (m, 45, arom. $\mathrm{C} \underline{\mathrm{H}}$ ), 7.50 (m, 30 , arom. $\underline{\mathrm{CH}}) .{ }^{13} \mathrm{C} \mathrm{NMR}\left(\mathrm{CDCl}_{3}, 100 \mathrm{MHz}\right): \delta 171.84$ (ㅁO), $137.93(\mathrm{C}), 129.13(\underline{\mathrm{CH}}), 128.34(\underline{\mathrm{C}} \mathrm{H})$, $126.09(\underline{\mathrm{CH}}), 101.12(\underline{\mathrm{CH}}), 70.41\left(\underline{\mathrm{CH}}_{2}\right), 69.28\left(\underline{\mathrm{CH}}_{2}\right), 68.93\left(\underline{\mathrm{CH}_{2}}\right), 66.32(\underline{\mathrm{CH}}), 63.85\left(\underline{\mathrm{CH}}_{2}\right), 62.25$ $\left(\underline{\mathrm{CH}}_{2}\right), 29.08\left(\underline{\mathrm{CH}}_{2}\right), 28.76\left(\underline{\mathrm{CH}}_{2}\right)$. MALDI-TOF Mw: 19548, Mn: 19506. SEC Mw: 15287, Mn: 14315, PDI: $1.07 . \mathrm{T}_{\mathrm{m}}: 49.9$.

\section{Synthesis of ([G3]-PGLSA-OH $)_{2}-$ PEG $_{1000}(\mathbf{1 0 b})-([G 3]-P G L S A-b z l d)_{2}-$ PEG $_{1000}(3.21$ g, 0.164} mmol) was dissolved in THF (30 mL) before adding $10 \%$ wt $\mathrm{Pd} / \mathrm{C}$. The solution was shaken under $\mathrm{H}_{2}$ at 60 psi overnight. The product was filtered over celite and the concentrated solution was precipitated in cold ethyl ether $(1 \mathrm{~L})$ to afford a white powder $\left(93 \%\right.$ yield). ${ }^{1} \mathrm{H} \mathrm{NMR}\left(\mathrm{CDCl}_{3}, 400 \mathrm{MHz}\right): \delta 2.64$ (broad m,

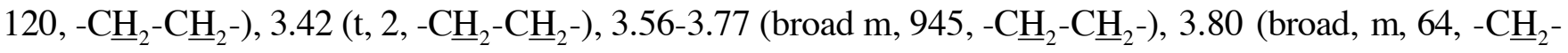
$\left.\mathrm{C}_{2_{2}}\right)$, 4.19-4.28 (m, 56, $\left.-\mathrm{C}_{2}-\mathrm{C}_{2}{ }^{-}\right), 4.88\left(\mathrm{~m}, 16,-\mathrm{CH}_{2}-\mathrm{C} \underline{\mathrm{H}}-\mathrm{CH}_{2}-\right), 5.23\left(\mathrm{~m}, 14,-\mathrm{CH}_{2}-\mathrm{C}_{1}-\mathrm{CH}_{2}-\right) .{ }^{13} \mathrm{C}$ NMR $\left(\mathrm{CDCl}_{3}, 100 \mathrm{MHz}\right): \delta 172.62$ (COOR), $70.31\left(\mathrm{CH}_{2}\right), 69.43\left(\mathrm{CH}_{2}\right), 69.06\left(\mathrm{CH}_{2}\right), 65.68(\mathrm{CH}), 63.97$ 
$\left(\mathrm{CH}_{2}\right), 63.33\left(\mathrm{CH}_{2}\right), 62.45\left(\mathrm{CH}_{2}\right), 64.22\left(\mathrm{CH}_{2}\right), 28.94\left(\mathrm{CH}_{2}\right)$. MALDI-TOF Mw: 18042, Mn: 17998. SEC Mw: 14358, Mn: 13451, PDI: 1.07. $\mathrm{T}_{\mathrm{m}}: 48.9$.

\section{Synthesis of ([G4]-PGLSA-bzld) $)_{2}-$ PEG $_{1000}(\mathbf{1 1 b})$ - This was prepared from ([G3]-PGLSA-OH) $)_{2}$ -} PEG $_{10000}(1.79 \mathrm{~g}, 0.0986 \mathrm{mmol})$ and $128 \mathrm{eq}$ of succinic acid mono-(2-phenyl-[1,3]dioxan-5-yl)ester anhydride $(6.85 \mathrm{~g}, 12.62 \mathrm{mmol})$ and $25.6 \mathrm{eq}$ of DMAP $(0.308 \mathrm{~g}, 2.525 \mathrm{mmol})$ while stirring for three days in DCM (30 mL). Any remaining anhydride was quenched with n-butanol $(1 \mathrm{~mL})$ and allowed to stir for another five hours. The resulting solution was washed with $0.1 \mathrm{~N} \mathrm{HCl}(200 \mathrm{~mL})$, saturated sodium bicarbonate $(3 \times 200 \mathrm{~mL})$ and brine $(200 \mathrm{~mL})$. The organic phase was dried with sodium sulfate before the solution was concentrated under reduced pressure. A white powder was collected by filtration after precipitation in cold $\left(-20^{\circ} \mathrm{C}\right)$ ethyl ether $\left(89 \%\right.$ yield). ${ }^{1} \mathrm{H} \mathrm{NMR}\left(\mathrm{CDCl}_{3}, 400 \mathrm{MHz}\right): \delta 2.61$ (broad m, 180, $-\underline{\mathrm{CH}}_{2}-\underline{\mathrm{CH}}_{2}-$ ), 2.71 (broad m, $70-\mathrm{C}_{2}-\underline{\mathrm{CH}}_{2}-$ ), 3.44 (t, 2, $-\underline{\mathrm{CH}}_{2}-\underline{\mathrm{CH}}_{2}-$ ), 3.57-3.66 (broad m, 936, - $\underline{\mathrm{H}}_{2}-\mathrm{C}_{2}{ }_{2}^{-}$),

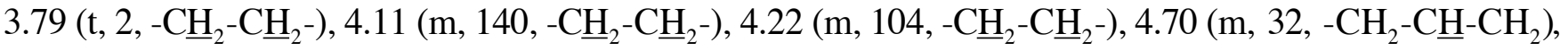
$5.22\left(\mathrm{~m}, 34,-\mathrm{CH}_{2}-\mathrm{C}_{\mathbf{H}}-\mathrm{CH}_{2}-\right), 5.51(\mathrm{~s}, 32, \mathrm{C} \underline{\mathrm{H}}), 7.33$ (m, 90, arom. $\left.\mathrm{C} \underline{\mathrm{H}}\right), 7.46$ (m, 60, arom. $\left.\mathrm{C} \underline{\mathrm{H}}\right) .{ }^{13} \mathrm{C}$

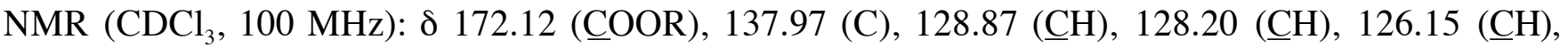
$100.98(\underline{\mathrm{CH}}), 71.70\left(\underline{\mathrm{CH}}_{2}\right), 70.46(\underline{\mathrm{CH}}), 69.13\left(\underline{\mathrm{CH}}_{2}\right), 66.45(\underline{\mathrm{CH}}), 62.34\left(\underline{\mathrm{CH}}_{2}\right), 29.30\left(\underline{\mathrm{CH}}_{2}\right), 29.16\left(\underline{\mathrm{CH}}_{2}\right)$, $28.80\left(\underline{\mathrm{CH}}_{2}\right)$. MALDI-TOF N/A. SEC Mw: 18456, Mn: 16352, PDI: 1.20. T $\mathrm{T}_{\mathrm{m}}: 42.5$.

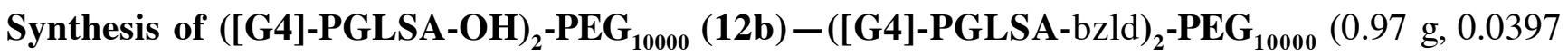
mmol) was dissolved in THF ( $5 \mathrm{~mL}$ ) before adding $10 \%$ wt $\mathrm{Pd} / \mathrm{C}$. The solution was shaken under $\mathrm{H}_{2}$ at 60 psi overnight. The product was filtered over celite and the concentrated solution was precipitated in cold ethyl ether $(1 \mathrm{~L})$ to afford a white powder (90\% yield). ${ }^{1} \mathrm{H} \mathrm{NMR}\left(\mathrm{CDCl}_{3}, 400 \mathrm{MHz}\right)$ : $\delta 2.63$ (broad m, 260, $-\underline{\mathrm{CH}}_{2}-\underline{\mathrm{CH}}_{2}-$ ), 3.57 (t, 2, - $\underline{\mathrm{H}}_{2}-\mathrm{C}_{\mathrm{H}_{2}}$ ), 3.60-3.67 (broad m, 921, - $\underline{\mathrm{H}}_{2}-\mathrm{C}_{\mathrm{H}_{2}}$ ), 3.78 (broad, m, 20, - $\underline{\mathrm{C}}_{2}-$

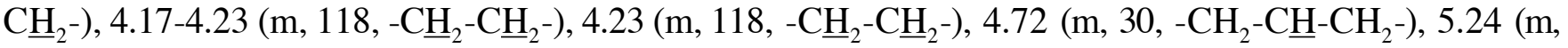

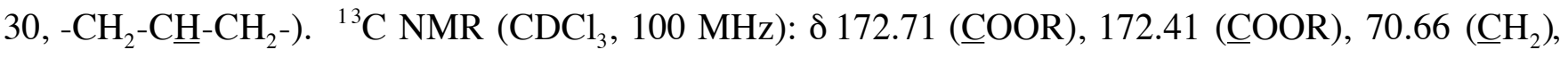


$70.04\left(\underline{\mathrm{CH}}_{2}\right), 65.76(\underline{\mathrm{CH}}), 63.35\left(\underline{\mathrm{CH}}_{2}\right), 29.04\left(\underline{\mathrm{CH}}_{2}\right)$. MALDI-TOF Mw: 22069, Mn: 21956. SEC Mw: 17834, Mn: 15680, PDI: 1.23. $\mathrm{T}_{\mathrm{m}}: 42.6$.

Synthesis of ([G0]-PGLSA-bzld) $)_{\mathbf{2}} \mathbf{P E G}_{\mathbf{3 4 0 0}} \mathbf{( 3 a )}$ - This was prepared from dry PEG $_{3400}(5.00 \mathrm{~g}, 1.47$ $\mathrm{mmol})$ with the addition of 8 eq of succinic acid mono-(2-phenyl-[1,3]dioxan-5-yl)ester anhydride (6.38 $\mathrm{g}$, $11.76 \mathrm{mmol})$ and $1.6 \mathrm{eq}$ of DMAP $(0.287 \mathrm{~g}, 2.35 \mathrm{mmol})$ while stirring overnight in DCM (40 mL). Any remaining anhydride was quenched with n-butanol $(1 \mathrm{~mL})$ and allowed to stir for another five hours. The resulting solution was washed with $0.1 \mathrm{~N} \mathrm{HCl}(200 \mathrm{~mL})$, saturated sodium bicarbonate $(3 \times 200 \mathrm{~mL})$ and brine $(200 \mathrm{~mL})$. The organic phase was dried with sodium sulfate before the solution was concentrated under reduced pressure. A white powder was collected by filtration after precipitation in cold $\left(-20{ }^{\circ} \mathrm{C}\right)$ ethyl ether (91\% yield). ${ }^{1} \mathrm{H} \mathrm{NMR}\left(\mathrm{CDCl}_{3}, 400 \mathrm{MHz}\right)$ : $\delta 2.67$ (broad m, 4, $\left.-\mathrm{C}_{2}-\mathrm{C}_{2}-\right)$, 2.74 (broad m, 4, -

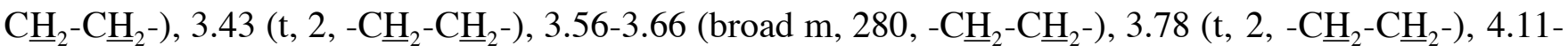

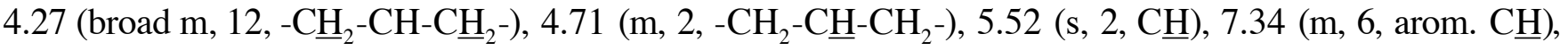

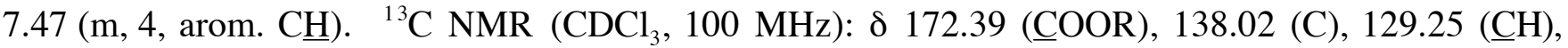
$\left.128.47(\underline{\mathrm{CH}}), 126.21(\underline{\mathrm{CH}}), 101.37(\underline{\mathrm{CH}}), 70.57\left(\underline{\mathrm{CH}}_{2}\right), 69.18(\underline{\mathrm{CH}})_{2}\right), 66.43(\underline{\mathrm{CH}}), 64.07\left(\underline{\mathrm{CH}_{2}}\right), 29.49$ $\left(\underline{\mathrm{CH}}_{2}\right), 29.21\left(\underline{\mathrm{CH}}_{2}\right)$. MALDI-TOF Mw: 3960, Mn: 3875, PDI: 1.02. SEC Mw: 3880, Mn: 3750, PDI: 1.04. $\mathrm{T}_{\mathrm{m}}=44.7$.

\section{Synthesis of ([G0]-PGLSA-OH $)_{\mathbf{2}}-\mathbf{P E G}_{\mathbf{3 4 0 0}}(\mathbf{4 a})-([G 0]-P G L S A-b z l d)_{\mathbf{2}}-\mathbf{P E G}_{\mathbf{3 4 0 0}}(4.98 \mathrm{~g}, 1.28$}

mmol) was dissolved in THF (50 mL) before adding $10 \%$ wt $\mathrm{Pd} / \mathrm{C}$. The solution was shaken under $\mathrm{H}_{2}$ at 60 psi overnight. The product was filtered over celite and the concentrated solution was precipitated in cold ethyl ether $(1 \mathrm{~L})$ to afford a white powder $\left(97 \%\right.$ yield). ${ }^{1} \mathrm{H} \mathrm{NMR}\left(\mathrm{CDCl}_{3}, 400 \mathrm{MHz}\right): \delta 2.61$ (broad m, 8, $\mathrm{C}_{\mathrm{H}_{2}}-\underline{\mathrm{CH}}_{2}-$ ), 3.38 (t, 2, - $\mathrm{C}_{2}-\underline{\mathrm{CH}}_{2}-$ ), 3.44-3.63 (broad m, 278, - $\underline{\mathrm{C}}_{2}-\underline{\mathrm{CH}}_{2}-$ ), 3.69 (broad m, 8, - $\underline{\mathrm{H}}_{2}-\mathrm{CH}-$ $\mathrm{CH}_{2}-$ ), 4.17 (broad m, 4, $\left.-\mathrm{CH}_{2}-\mathrm{CH}-\mathrm{CH}_{2}-\right), 4.85$ (m, 2, $\left.-\mathrm{CH}_{2}-\mathrm{CH}-\mathrm{CH}_{2}-\right)$. ${ }^{13} \mathrm{C} \mathrm{NMR}\left(\mathrm{CDCl}_{3}, 100 \mathrm{MHz}\right): \delta$

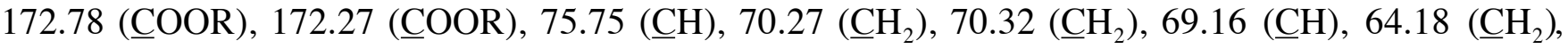


$62.06\left(\underline{\mathrm{CH}}_{2}\right), 29.66\left(\underline{\mathrm{CH}}_{2}\right), 29.59\left(\underline{\mathrm{CH}}_{2}\right)$. MALDI-TOF Mw: 3769, Mn: 3696, PDI: 1.02. SEC Mw: 3640, Mn: 3500, PDI: 1.04. $\mathrm{T}_{\mathrm{m}}=46.6$.

\section{Synthesis of ([G1]-PGLSA-bzld $)_{2}-\mathbf{P E G}_{\mathbf{3 4 0 0}}(\mathbf{5 a})$ - This was prepared from ([G0]-PGLSA-OH) $)_{2}$ -} PEG $_{3400}$ (4.33 g, 1.166 mmol), 16 eq of succinic acid mono-(2-phenyl-[1,3]dioxan-5-yl)ester anhydride (10.12 g, $18.65 \mathrm{mmol})$ and $3.2 \mathrm{eq}$ of DMAP (0.456 g, $3.73 \mathrm{mmol})$ and allowed to stir overnight in DCM $(50 \mathrm{~mL})$. Any remaining anhydride was quenched with $\mathrm{n}$-butanol $(1 \mathrm{~mL})$ and allowed to stir for another five hours. The resulting solution was washed with $0.1 \mathrm{~N} \mathrm{HCl}(200 \mathrm{~mL})$, saturated sodium bicarbonate (3 x $200 \mathrm{~mL}$ ) and brine $(200 \mathrm{~mL})$. The organic phase was dried with sodium sulfate before the solution was concentrated under reduced pressure. A white powder was collected by filtration after precipitation in cold $\left(-20{ }^{\circ} \mathrm{C}\right.$ ) ethyl ether (93\% yield). ${ }^{1} \mathrm{H} \mathrm{NMR}\left(\mathrm{CDCl}_{3}, 400 \mathrm{MHz}\right): \delta 2.55$ (broad m, 16, $\left.-\mathrm{CH}_{2}-\mathrm{CH}_{2}-\right), 2.65$ (broad m, 8, $-\underline{\mathrm{CH}}_{2}-\mathrm{C}_{2}-$ ), 3.36 (t, 2, $-\underline{\mathrm{CH}}_{2}-\mathrm{C}_{2}-$ ), 3.53-3.63 (broad m, 282, $-\mathrm{C}_{2}-\underline{\mathrm{CH}}_{2}-$ ), 3.71 (t, 2, - $\underline{\mathrm{C}}_{2}-$ $\mathrm{C}_{2}{ }^{-}$), 4.05 (broad m, 12, $-\mathrm{C}_{2}-\mathrm{CH}-\mathrm{CH}_{2}-$ ), 4.13 (broad m, 14, - $\underline{\mathrm{CH}}_{2}-\mathrm{CH}-\mathrm{C}_{2}{ }^{-}$), 4.62 (m, 4, - $\mathrm{CH}_{2}-\mathrm{C} \underline{\mathrm{H}}-$ $\left.\mathrm{CH}_{2}{ }^{-}\right), 5.13$ (m, 2, - $\left.\mathrm{CH}_{2}-\mathrm{C}_{\underline{H}}-\mathrm{CH}_{2}-\right), 5.43$ (s, 4, $\underline{\mathrm{H}}$ ), 7.26 (m, 12, arom. $\mathrm{C} \underline{\mathrm{H}}$ ), 7.39 (m, 8, arom. $\mathrm{C} \underline{\mathrm{H}}$ ). ${ }^{13} \mathrm{C}$

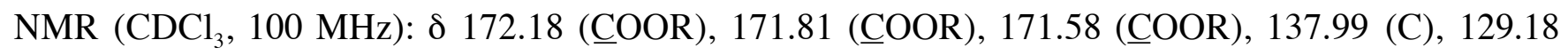
$(\underline{\mathrm{CH}}), 128.40(\underline{\mathrm{CH}}), 126.16(\underline{\mathrm{CH}}), 101.22(\underline{\mathrm{CH}}), 70.70\left(\underline{\mathrm{CH}}_{2}\right), 69.35(\underline{\mathrm{CH}}), 69.15\left(\underline{\mathrm{CH}}_{2}\right), 66.47(\underline{\mathrm{CH}}), 64.00$ $\left(\underline{\mathrm{CH}}_{2}\right), 62.42\left(\underline{\mathrm{CH}}_{2}\right), 29.30\left(\underline{\mathrm{CH}}_{2}\right), 29.08\left(\underline{\mathrm{CH}}_{2}\right), 28.99\left(\underline{\mathrm{CH}}_{2}\right)$. MALDI MS Mw: 4844, Mn: 4749, PDI: 1.02. SEC Mw: 3950, Mn: 3790, PDI: $1.04 . \mathrm{T}_{\mathrm{m}}=38.8$.

\section{Synthesis of ([G1]-PGLSA-OH $)_{2}-$ PEG $_{\mathbf{3 4 0 0}}(\mathbf{6 a})-([\mathbf{G 1}]-\mathbf{P G L S A - b z l d})_{2}-\mathbf{P E G}_{\mathbf{3 4 0 0}}(4.64 \mathrm{~g}, 0.974$}

mmol) was dissolved in THF (30 mL) before adding $10 \% \mathrm{wt} \mathrm{Pd} / \mathrm{C}$. The solution was shaken under $\mathrm{H}_{2}$ at 60 psi overnight. The product was filtered over celite and the concentrated solution was precipitated in cold ethyl ether (1 L) to afford a white powder (93\% yield). ${ }^{1} \mathrm{H} \mathrm{NMR}\left(\mathrm{CDCl}_{3}, 400 \mathrm{MHz}\right): \delta 2.63$ (broad m, 24, $-\mathrm{CH}_{2}-\underline{\mathrm{CH}}_{2}-$ ), 3.42 (t, 2, - $\underline{\mathrm{H}}_{2}-\underline{\mathrm{CH}}_{2}-$ ), 3.50-3.67 (broad m, 278, - $\mathrm{C}_{2_{2}}-\underline{\mathrm{CH}}_{2}-$ ), 3.76 (broad m, 15, - $\mathrm{CH}_{2}-\mathrm{CH}-$ $\mathrm{C}_{2}{ }^{-}$), 4.14-4.29 (broad m, 12, $-\mathrm{C}_{2}-\mathrm{CH}-\mathrm{CH}_{2}-$ ), 4.89 (m, 4, - $\mathrm{CH}_{2}-\mathrm{C} \underline{\mathrm{H}}-\mathrm{CH}_{2}-$ ), 5.24 (m, 2, - $\mathrm{CH}_{2}-\mathrm{C}_{\mathbf{H}}-\mathrm{CH}_{2}$ ). ${ }^{13} \mathrm{C}$ NMR $\left(\mathrm{CDCl}_{3}, 100 \mathrm{MHz}\right): \delta 172.41$ (ㅁO), $75.86(\underline{\mathrm{C}} \mathrm{H}), 70.76\left(\underline{\mathrm{CH}_{2}}\right), 70.19(\underline{\mathrm{C}} \mathrm{H}), 69.21\left(\underline{\mathrm{CH}}_{2}\right)$, $65.89(\underline{\mathrm{CH}}), 64.13\left(\underline{\mathrm{CH}}_{2}\right), 63.51\left(\underline{\mathrm{CH}}_{2}\right), 62.56\left(\underline{\mathrm{CH}}_{2}\right), 62.08\left(\underline{\mathrm{CH}}_{2}\right), 29.48\left(\underline{\mathrm{CH}}_{2}\right), 29.37\left(\underline{\mathrm{CH}}_{2}\right), 29.21$ 
$\left(\underline{\mathrm{CH}}_{2}\right), 29.10\left(\underline{\mathrm{CH}}_{2}\right)$. MALDI MS Mw: 4487, Mn: 4394, PDI: 1.02. SEC Mw: 4590, Mn: 4440, PDI: 1.03. $\mathrm{T}_{\mathrm{m}}=41.9$.

\section{Synthesis of ([G2]-PGLSA-bzld $)_{2}-\mathbf{P E G}_{\mathbf{3 4 0 0}}(\mathbf{7 a})$ - This was prepared from ([G1]-PGLSA-OH) $)_{2}$ -} PEG $_{3400}(3.25 \mathrm{~g}, 0.737 \mathrm{mmol})$ and $32 \mathrm{eq}$ of succinic acid mono-(2-phenyl-[1,3]dioxan-5-yl)ester anhydride (12.79 g, $23.58 \mathrm{mmol})$ and $6.4 \mathrm{eq}$ of DMAP $(0.576 \mathrm{~g}, 4.72 \mathrm{mmol})$. The reaction was stirred overnight in DCM (50 mL). Any remaining anhydride was quenched with n-butanol $(1 \mathrm{~mL})$ and allowed to stir for another five hours. The resulting solution was washed with $0.1 \mathrm{~N} \mathrm{HCl}(200 \mathrm{~mL})$, saturated sodium bicarbonate $(3 \times 200 \mathrm{~mL})$ and brine $(200 \mathrm{~mL})$. The organic phase was dried with sodium sulfate before the solution was concentrated under reduced pressure. A white powder was collected by filtration after precipitation in cold $\left(-20{ }^{\circ} \mathrm{C}\right)$ ethyl ether $(91 \%$ yield $) .{ }^{1} \mathrm{H} \mathrm{NMR}\left(\mathrm{CDCl}_{3}, 400 \mathrm{MHz}\right): \delta 2.61$ (broad m, 40, - $\underline{\mathrm{C}}_{2}-\underline{\mathrm{C}}_{2}-$ ), 2.72 (broad m, 16, - $\underline{\mathrm{C}}_{2}-\underline{\mathrm{C}}_{2}-$ ), 3.43 (t, 2, - $\underline{\mathrm{CH}}_{2}-\underline{\mathrm{C}}_{2}{ }^{-}$), 3.55-3.65 (broad m, 280, - $\underline{\mathrm{C}}_{2}-$

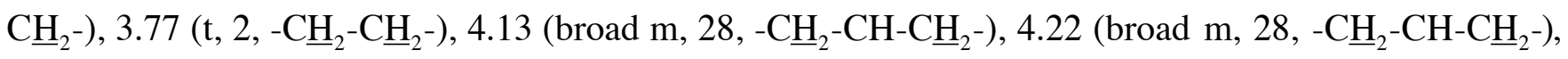

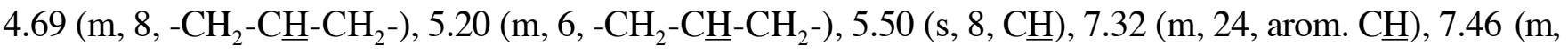

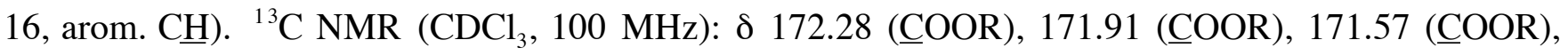
$138.01(\mathrm{C}), 129.26(\underline{\mathrm{CH}}), 128.48(\underline{\mathrm{CH}}), 126.21(\underline{\mathrm{CH}}), 101.33(\underline{\mathrm{CH}}), 70.56\left(\underline{\mathrm{CH}}_{2}\right), 69.50(\underline{\mathrm{C}} \mathrm{H}), 69.16$ $\left(\underline{\mathrm{CH}}_{2}\right), 66.53(\underline{\mathrm{CH}}), 64.08\left(\underline{\mathrm{CH}}_{2}\right), 29.49\left(\underline{\mathrm{CH}}_{2}\right), 29.21\left(\underline{\mathrm{CH}}_{2}\right)$. MALDI MS Mw: 6642, Mn: 6492, PDI: 1.02. SEC Mw: 4860, Mn: 4680, PDI: $1.04 . \mathrm{T}_{\mathrm{m}}=31.4$.

\section{Synthesis of ([G2]-PGLSA-OH) $)_{\mathbf{2}}-$ PEG $_{\mathbf{3 4 0 0}}$ (8a)-([G2]-PGLSA-bzld) $)_{\mathbf{2}}-\mathbf{P E G}_{\mathbf{3 4 0 0}}(3.03 \mathrm{~g}, 0.466$} mmol) was dissolved in THF (30 mL) before adding $10 \%$ wt $\mathrm{Pd} / \mathrm{C}$. The solution was shaken under $\mathrm{H}_{2}$ at 60 psi overnight. The product was filtered over celite and the concentrated solution was precipitated in cold ethyl ether $(1 \mathrm{~L})$ to afford a white powder $\left(98 \%\right.$ yield). ${ }^{1} \mathrm{H} \mathrm{NMR}\left(\mathrm{CDCl}_{3}, 400 \mathrm{MHz}\right)$ : $\delta 2.63$ (broad m, 56, $-\mathrm{CH}_{2}-\mathrm{CH}_{2}-$ ), 3.42 (s, 4, - $\mathrm{CH}_{2}-\mathrm{CH}_{2}-$ ), 3.50-3.67 (broad m, 285, - $\mathrm{C}_{2_{2}}-\underline{\mathrm{CH}}_{2}-$ ), 3.72 (broad m, 27, - $\mathrm{CH}_{2}-\mathrm{CH}-$

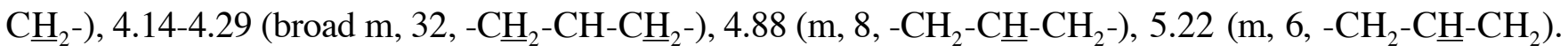

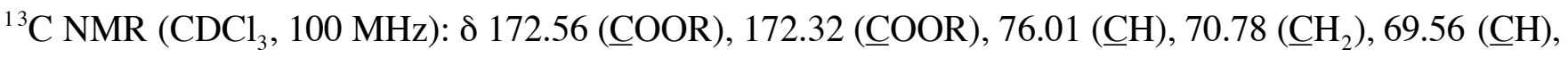


$69.22\left(\underline{\mathrm{CH}}_{2}\right), 64.14\left(\underline{\mathrm{CH}}_{2}\right), 63.52\left(\underline{\mathrm{CH}}_{2}\right), 62.60\left(\underline{\mathrm{CH}}_{2}\right), 61.93\left(\underline{\mathrm{CH}}_{2}\right), 29.44\left(\mathrm{CH}_{2}\right), 29.21\left(\mathrm{CH}_{2}\right), 28.98$

$\left(\underline{\mathrm{CH}}_{2}\right)$. MALDI MS Mw: 5910, Mn: 5788, PDI: 1.02. SEC Mw: 5340, Mn: 5210, PDI: 1.03. $\mathrm{T}_{\mathrm{m}}=36.5$.

\section{Synthesis of ([G3]-PGLSA-bzld) $)_{2}-\mathbf{P E G}_{3400}(\mathbf{9 a})$ - This was prepared from ([G2]-PGLSA-OH) $)_{2}$ -} $\mathbf{P E G}_{3400}(2.13 \mathrm{~g}, 0.367 \mathrm{mmol})$ and $64 \mathrm{eq}$ of succinic acid mono-(2-phenyl-[1,3]dioxan-5-yl)ester anhydride $(12.75 \mathrm{~g}, 23.49 \mathrm{mmol})$ and $12.8 \mathrm{eq}$ of DMAP $(0.574 \mathrm{~g}, 4.70 \mathrm{mmol})$. The reaction was stirred overnight in DCM $(50 \mathrm{~mL})$. Any remaining anhydride was quenched with n-butanol $(1 \mathrm{~mL})$ and allowed to stir for another five hours. The resulting solution was washed with $0.1 \mathrm{~N} \mathrm{HCl}(200 \mathrm{~mL})$, saturated sodium bicarbonate $(3 \times 200 \mathrm{~mL})$ and brine $(200 \mathrm{~mL})$. The organic phase was dried with sodium sulfate before the solution was concentrated under reduced pressure. A white powder was collected by filtration after precipitation in cold $\left(-20^{\circ} \mathrm{C}\right)$ ethyl ether (92\% yield). ${ }^{1} \mathrm{H}$ NMR $\left(\mathrm{CDCl}_{3}, 400 \mathrm{MHz}\right): \delta 2.61$ (broad m, 84, - $\mathrm{C}_{2}-\mathrm{C}_{2}-$ ), 2.74 (broad m, 36, - $\mathrm{C}_{2}-\mathrm{C}_{2}-$ ), 3.43 (t, 2, - $\underline{\mathrm{CH}}_{2}-\mathrm{C}_{2}-$ ), 3.56-3.65 (broad m, 278, - $\mathrm{C}_{2}-$ $\mathrm{C}_{2}-$ ), 3.78 (t, 2, - $\underline{\mathrm{CH}}_{2}-\mathrm{CH}_{2}-$ ), 4.13 (broad m, 60, - $\mathrm{C}_{2}-\mathrm{CH}-\mathrm{C}_{2}-$ ), 4.21 (broad m, 60, - $\underline{\mathrm{CH}}_{2}-\mathrm{CH}-\mathrm{CH}_{2}-$ ), 4.69 (m, 16, - $\left.\mathrm{CH}_{2}-\mathrm{C} \underline{\mathrm{H}}-\mathrm{CH}_{2}\right), 5.19$ (m, 14, - $\mathrm{CH}_{2}-\mathrm{C} \underline{\mathrm{H}}-\mathrm{CH}_{2}-$ ), 5.50 (s, 16, $\left.\mathrm{C} \underline{\mathrm{H}}\right), 7.32$ (m, 46, arom. $\mathrm{C} \underline{\mathrm{H}}$ ), 7.46

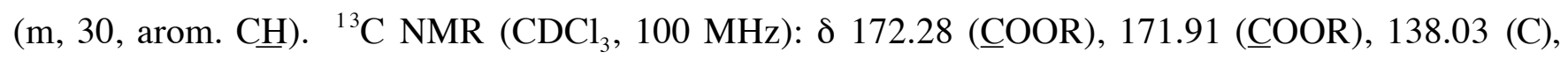
$129.26(\underline{\mathrm{C} H}), 128.48(\underline{\mathrm{C}} \mathrm{H}), 126.21(\underline{\mathrm{C}} \mathrm{H}), 101.31(\underline{\mathrm{CH}}), 70.76\left(\underline{\mathrm{CH}}_{2}\right), 69.49(\underline{\mathrm{C}} \mathrm{H}), 69.16\left(\underline{\mathrm{C}} \mathrm{H}_{2}\right), 66.53$ $(\underline{\mathrm{CH}}), 62.47\left(\underline{\mathrm{CH}}_{2}\right), 29.35\left(\underline{\mathrm{C}} \mathrm{H}_{2}\right), 29.02\left(\underline{\mathrm{CH}}_{2}\right), 28.83\left(\underline{\mathrm{CH}}_{2}\right)$. MALDI MS Mw: 10215, Mn: 9961, PDI: 1.02. SEC Mw: 7020, Mn: 6900, PDI: $1.02 . \mathrm{T}_{\mathrm{g}}=-13.6$.

\section{Synthesis of ([G3]-PGLSA-OH) $)_{2}-\mathbf{P E G}_{3400}(\mathbf{1 0 a})-([G 3]-P G L S A-b z l d)_{2}-\mathbf{P E G}_{\mathbf{3 4 0 0}}(2.35 \mathrm{~g}, 0.235$} mmol) was dissolved in THF $(50 \mathrm{~mL})$ before adding $10 \% \mathrm{wt} \mathrm{Pd} / \mathrm{C}$. The solution was shaken under $\mathrm{H}_{2}$ at 60 psi overnight. The product was filtered over celite and the concentrated solution was precipitated in cold ethyl ether $(1 \mathrm{~L})$ to afford a white powder $\left(98 \%\right.$ yield). ${ }^{1} \mathrm{H} \mathrm{NMR}\left(\left(\mathrm{CD}_{3}\right)_{2} \mathrm{CO}, 400 \mathrm{MHz}\right): \delta 2.64$ (broad m, 120, - $\mathrm{C}_{2}-\mathrm{C}_{2}{ }^{-}$), 3.49-3.60 (broad m, 286, - $\mathrm{C}_{2}-\mathrm{C}_{2}-$ ), 3.64-3.75 (broad m, 33, - $\mathrm{C}_{2}-\mathrm{CH}-\mathrm{C}_{2}{ }^{-}$), 4.004.12 (broad m, 42, - $\underline{\mathrm{CH}}_{2}-\mathrm{CH}-\mathrm{C}_{2}-$ ), 4.13-4.29 (broad m, 68, - $\mathrm{CH}_{2}-\mathrm{C} \underline{\mathrm{H}}-\mathrm{CH}_{2}-$ ), 4.64 (t, 2, - $\mathrm{CH}_{2}-\mathrm{C}^{-}-\mathrm{CH}_{2}-$ ), $4.85\left(\mathrm{t}, 2,-\mathrm{CH}_{2}-\mathrm{C} \underline{\mathrm{H}}-\mathrm{CH}_{2}-\right), 5.26\left(\mathrm{~m}, 14,-\mathrm{CH}_{2}-\mathrm{C} \underline{\mathrm{H}}-\mathrm{CH}_{2}-\right) .{ }^{13} \mathrm{C} \mathrm{NMR}\left(\left(\mathrm{CD}_{3}\right)_{2} \mathrm{CO}, 100 \mathrm{MHz}\right): \delta 171.85$ (ㅁOR), $171.64(\underline{\mathrm{COOR}}), 76.09(\underline{\mathrm{CH}}), 73.70\left(\underline{\mathrm{C}} \mathrm{H}_{2}\right), 70.56(\underline{\mathrm{CH}}), 69.52\left(\underline{\mathrm{CH}}_{2}\right), 66.19(\underline{\mathrm{C}} \mathrm{H}), 63.87\left(\underline{\mathrm{CH}}_{2}\right)$, 
$62.31\left(\underline{\mathrm{CH}}_{2}\right), 61.65\left(\underline{\mathrm{CH}}_{2}\right), 60.69\left(\underline{\mathrm{CH}}_{2}\right)$. MALDI MS Mw: 8765, Mn: 8575, PDI: 1.02. SEC Mw: 8090, Mn: 7820, PDI: 1.03. $\mathrm{T}_{\mathrm{g}}=-38.2$.

\section{Synthesis of ([G4]-PGLSA-bzld $)_{2}$-PEG $_{\mathbf{3 4 0 0}}$ (11a) - This was prepared from ([G3]-PGLSA-OH) $)_{2}$ -} PEG $_{3400}(1.82 \mathrm{~g}, 0.212 \mathrm{mmol})$ and $128 \mathrm{eq}$ of succinic acid mono-(2-phenyl-[1,3]dioxan-5-yl)ester anhydride (14.72 g, $27.12 \mathrm{mmol})$ and $25.6 \mathrm{eq}$ of DMAP $(0.663 \mathrm{~g}, 5.42 \mathrm{mmol})$ while stirring overnight in THF (50 mL). Any remaining anhydride was quenched with n-butanol (1 mL) and allowed to stir for another five hours. The resulting solution was washed with $0.1 \mathrm{~N} \mathrm{HCl}(200 \mathrm{~mL})$, saturated sodium bicarbonate $(3 \times 200 \mathrm{~mL})$ and brine $(200 \mathrm{~mL})$. The organic phase was dried with sodium sulfate before the solution was concentrated under reduced pressure. A white powder was collected by filtration after precipitation in cold $\left(-20^{\circ} \mathrm{C}\right)$ ethyl ether (87\% yield). ${ }^{1} \mathrm{H} \mathrm{NMR}\left(\mathrm{CDCl}_{3}, 400 \mathrm{MHz}\right): \delta 2.61$ (broad m, 180, $-\underline{\mathrm{CH}}_{2}-\mathrm{C}_{2}-$ ), 2.70 (broad m, 64, - $\underline{\mathrm{CH}}_{2}-\underline{\mathrm{CH}}_{2}-$ ), 3.43 (t, 2, - $\underline{\mathrm{H}}_{2}-\underline{\mathrm{CH}}_{2}-$ ), 3.56-3.65 (broad m, 286, - $\underline{\mathrm{H}}_{2}-\mathrm{C}_{2}$ ), 3.78 (t, 2, $-\mathrm{CH}_{2}-\mathrm{CH}_{2}-$ ), 4.11 (broad m, 125, $-\mathrm{CH}_{2}-\mathrm{CH}-\mathrm{CH}_{2}-$ ), 4.23 (broad m, 125, - $\mathrm{CH}_{2}-\mathrm{CH}-\mathrm{CH}_{2}-$ ), 4.68

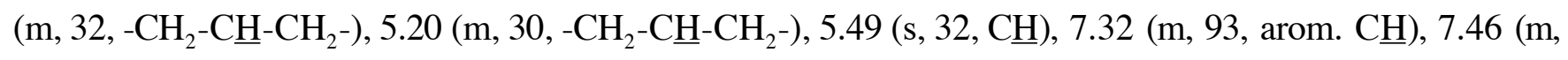

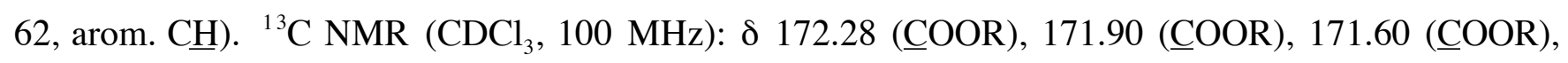
$138.04(\mathrm{C}), 129.26(\underline{\mathrm{CH}}), 128.48(\underline{\mathrm{CH}}), 126.21(\underline{\mathrm{CH}}), 101.29(\underline{\mathrm{CH}}), 70.76\left(\underline{\mathrm{CH}}_{2}\right), 69.46(\underline{\mathrm{C}} \mathrm{H}), 69.15$ $\left(\underline{\mathrm{CH}}_{2}\right), 66.53(\underline{\mathrm{CH}}), 62.57\left(\underline{\mathrm{CH}}_{2}\right), 29.34\left(\underline{\mathrm{CH}}_{2}\right), 29.18\left(\underline{\mathrm{CH}}_{2}\right), 29.02\left(\underline{\mathrm{CH}}_{2}\right), 28.83\left(\underline{\mathrm{CH}}_{2}\right)$. MALDI MS Mw: 17289, Mn: 16968, PDI: 1.02. SEC Mw: 8110, Mn: 7950, PDI: 1.02. $\mathrm{T}_{\mathrm{g}}=5.3$.

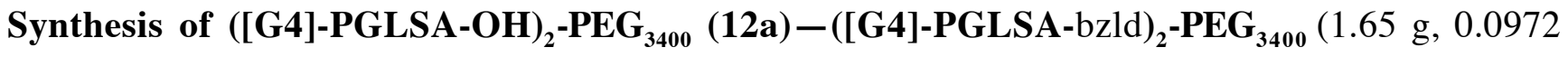
mmol) was dissolved in THF (15 mL) before adding $10 \%$ wt $\mathrm{Pd} / \mathrm{C}$. The solution was shaken under $\mathrm{H}_{2}$ at 60 psi overnight. The product was filtered over celite and the concentrated solution was precipitated in cold ethyl ether $(1 \mathrm{~L})$ to afford a white powder $\left(98 \%\right.$ yield). ${ }^{1} \mathrm{H} \mathrm{NMR}\left(\left(\mathrm{CD}_{3}\right)_{2} \mathrm{CO}, 400 \mathrm{MHz}\right): \delta 2.64$ (broad m, 248, $-\mathrm{C}_{2}-\underline{\mathrm{CH}}_{2}-$ ), 3.49-3.60 (broad m, 296, $-\mathrm{C}_{2}-\mathrm{CH}_{2}-$ ), 3.66 (broad m, 50, - $\mathrm{CH}_{2}-\mathrm{CH}-\mathrm{CH}_{2}-$ ), 3.82 (broad m, 42, $-\underline{\mathrm{CH}}_{2}-\mathrm{CH}-\underline{\mathrm{C}}_{2}-$ ), 4.04-4.16 (broad m, 66, - $\underline{\mathrm{C}}_{2}-\mathrm{C} \underline{\mathrm{H}}-\underline{\mathrm{C}}_{2}-$ ), 4.28 (broad m, 124, - $\underline{\mathrm{H}}_{2}-\mathrm{C} \underline{\mathrm{H}}_{-}-\underline{\mathrm{C}}_{2}$ ), $4.86\left(\mathrm{~m}, 10,-\mathrm{CH}_{2}-\mathrm{C}_{-}-\mathrm{CH}_{2}-\right), 5.27\left(\mathrm{~m}, 30,-\mathrm{CH}_{2}-\mathrm{C} \underline{\mathrm{H}}-\mathrm{CH}_{2}-\right) .{ }^{13} \mathrm{C} \mathrm{NMR}\left(\left(\mathrm{CD}_{3}\right)_{2} \mathrm{CO}, 100 \mathrm{MHz}\right): \delta 172.20$ 
(ㅁOR), $70.45\left(\underline{\mathrm{CH}}_{2}\right), 70.10(\underline{\mathrm{CH}}), 69.92\left(\underline{\mathrm{CH}}_{2}\right), 65.96(\underline{\mathrm{CH}}), 62.31\left(\underline{\mathrm{CH}}_{2}\right)$. MALDI MS Mw: 14402, Mn: 14146, PDI: 1.02. SEC Mw: 9130, Mn: 8990, PDI: 1.02. $\mathrm{T}_{\mathrm{g}}=-18.0$.
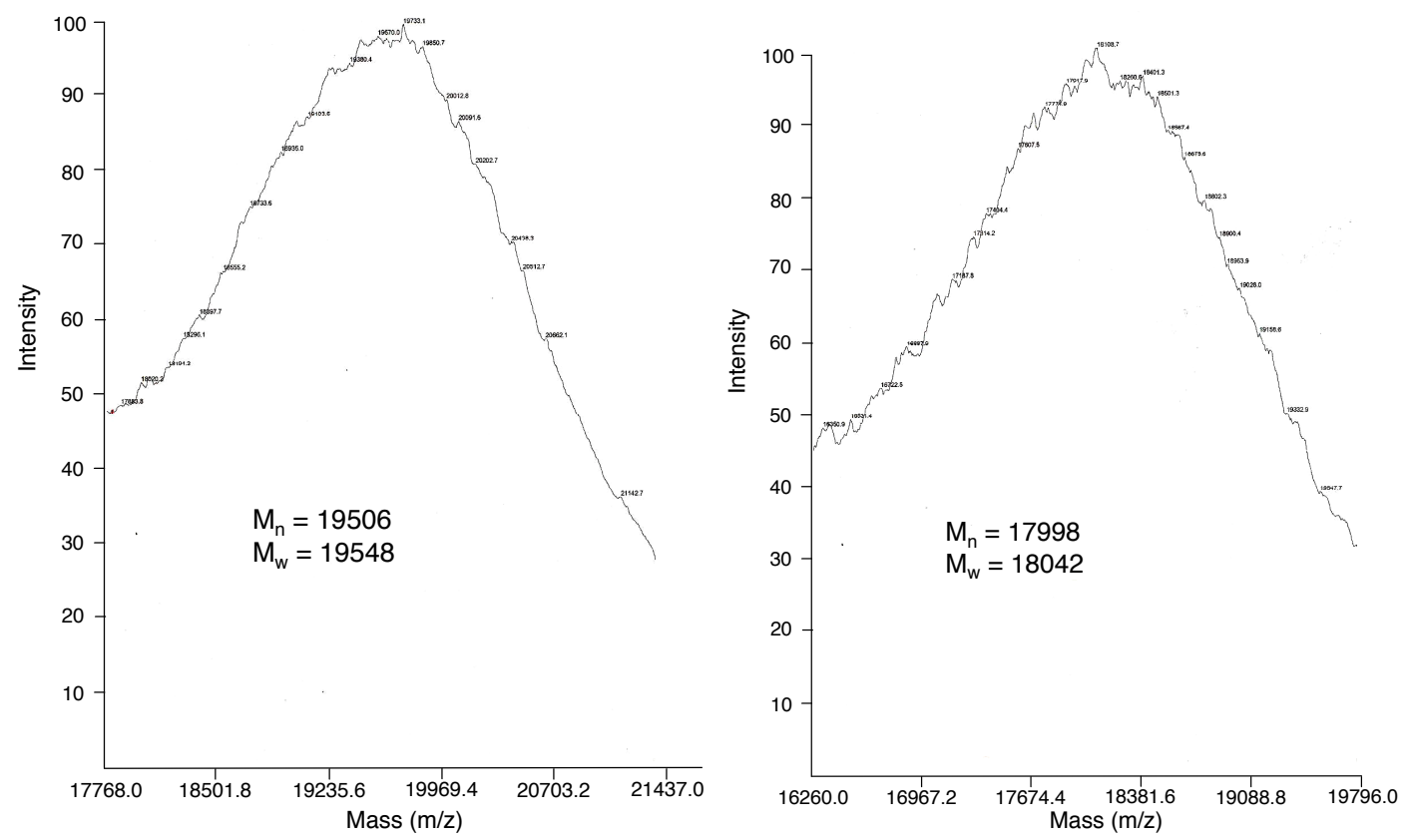

Figure S1. MALDI spectra of (left) ([G3]-PGLSA-bzld) -PEG $_{10000}$ and (right) ([G3]-PGLSA$\mathrm{OH})_{2} \mathbf{P E G}_{10000}$. 